\title{
Transcranial Focused Ultrasound Alters Conflict and Emotional Processing, Physiology, and Performance II: Right Anterior Insula/Frontal Operculum Targeting.
}

\author{
Maria Fini and William J. Tyler* \\ School of Biological and Health Systems Engineering \\ Arizona State University \\ Tempe, Arizona 85287 USA
}

\begin{abstract}
The right anterior insula/frontal operculum (aIns/fO) plays a key role in cognitive and state control as it is involved in attention, emotion, autonomic responses, and integrates saliency. It is also known to be active in mindfulness meditation, and is involved in interoceptive awareness, but also executive control, performance evaluation, and attentional maintenance. This study aimed to assess the feasibility of modulating cognitive control and emotional processing by targeting the aIns/fO with tFUS during a modified version of the Erikson flanker paradigm in which fearful and neutral faces were placed behind the flanker task as emotional distractors. Subjects were divided into two groups, one receiving neuro-navigated tFUS to the aIns/fO and the other receiving Sham. Our observations indicate that modulation of the aIns/fO with tFUS reduced parasympathetic fear responses seen in heart rate variability (HRV), emotional distraction interference on performance, and post-error slowing. Processing differences were measured across groups and demonstrated modulation of event related potentials associated with saliency, emotion, and congruency as well as modulation event-locked delta and beta with tFUS. These frequencies are known to be involved in modulation of physiologic responses though interaction with the amygdala, as well as distractor process through insular-medial frontal regulation during feedback. Overall, this study demonstrates that tFUS along with MR-neuronavigation can be used to target specific anatomical areas to induce larger network modulation producing changes in emotional attention, cognitive control, and conflict resolution.
\end{abstract}

Keywords: emotional regulation, mental health, default mode network, focused ultrasound, neuromodulation, neurotechnology. *Correspondence wtyler@asu.edu.

\section{INTRODUCTION}

The right anterior insula and frontal operculum (aIns/fO) likely plays an intriguing role in producing the contents of conscious experience; it is highly involved in both emotional expression and cognitive control, and may play a key role in directing present-center attention and plays a key role in mindfulness meditation (Tang et al., 2015). The functional area of the right aIns/fO spreads from the anterior insular cortex laterally through the inferior frontal operculum, and throughout the pars opercularis (BA44/45) of the right inferior frontal gyrus (rIFG) (Dosenbach et al., 2008). It is part of the cingularopercular network (Cai et al., 2014; Coste and Kleinschmidt, 2016), and the ventral attention network (Dosenbach et al., 2006).

The aIns/fO is involved in diverse cognitive functions related to attention, is thought to be critical for integrating saliency queues, and orchestrates switching between the default mode (DMN) and executive networks (Eckert et al., 2009; Sridharan et al., 2008). It is thought to integrate emotional salience with afferent homeostatic information from the posterior insula in a bilateral fashion (Craig, 2002; Craig, 2004). The anterior insula is also involved in communicating information about fear stimulus to the amygdala, an important component of controlling physiologic fear expression (Phelps et al., 2001; Shi and Davis, 1999; Williams et al., 2004). It is known to be involved in interoceptive awareness and volitional control of autonomic responses (Critchley et al., 2002b). The aIns/fO is associated with pain and emotion in both first and third person (Jabbi and Keysers, 2008), and is thought to code for negative valence (Corradi-Dell'Acqua et al., 2016). Some authors have proposed this region contributes to the perceptual experience of the body and internal emotional state (Critchley et al., 2004).

Activity in the aIns/fO specifically lateralized to the right is greatly implicated in cognitive control, and is highly connected to the subthalamic nucleus, allowing for broad cortical influence (Aron et al., 2016). The right aIns/fO is linked to attentional performance (Aron et al., 2014), monitoring, and evaluation (Eckert et al., 2009), as well as action conflict (Hampshire et al., 2010), inhibitory control (Cai et al., 2014; Levy and Wagner, 2011), environmental monitoring, response selection (Taylor et al., 2009 ; Zaki et al., 2012), and error processing (Iannaccone et al., 2015 ; Wessel and Aron, 2017; Wessel et al., 2012). It likely plays a key role in attentional maintenance (Dosenbach et al., 2008) through effortful perception (Wild et al., 2012), and in emotional distraction (Shafer et al., 2012). 
There is growing optimism in the field of neurostimulation that transcranial focused ultrasound (tFUS) can be used to modulate brain networks for the purpose of enhancing executive functioning and wellbeing (Fini and Tyler, 2017; Sanguinetti et al., 2020). The possibility of modulating executive control in response to cognitive interference and emotional distraction by targeting tFUS at the aIns/fO is explored here. tFUS was delivered in a trial-by-trial manner to the aIns/fO while subjects performed a modified version of the Erickson Flanker (Eriksen and Eriksen, 1974) task in which emotional faces (fear, neutral, or scrambled) were displayed in the background as distractors. The Flanker task has been widely used to study cognitive control and response to interference, and produces well studied evoked electroencephalogram (EEG) activity and error responses. For example, trial-locked frontocentral theta and delta EEG activity can be seen during conflict processing and posterror (Debener et al., 2005; Iannaccone et al., 2015). Others have demonstrated that preceding conflict processing task trials with images of emotional faces induces response slowing and recruits the cingulo-opercular network (Papazacharias et al., 2015).

Behavioral performance was measured by reaction times, accuracy, and conflict adaption. Heart rate data was collected for heart rate variability (HRV) analysis. Mood assessments were collected using the Positive and Negative Affect Scale (PANAS; Crawford and Henry, 2004).

\section{RESULTS}

Twenty-eight healthy participants were randomly divided into two groups: one group receiving sham, and the other receiving active tFUS targeted to the anterior insula and frontal operculum (aIns/fO) on each trial. All subjects provided informed consent and all procedures were approved by the Institutional Review Board at Arizona State University. The tFUS stimulation mean center MNI coordinates of $(x=35.4 \pm 13, y=13.5 \pm 1.2, z=1.4 \pm 1.7$, mean \pm SEM, Figure 1 , Table S1) were recorded. After performing a brief flanker baseline, the subjects were asked to perform a modified version of the Erikson flanker paradigm in which emotional faces were presented as distractors behind the flanker arrows (Figure S7). EEG, heart rate, reaction time, accuracy, and PANAS mood scale were recorded.

\section{The influence of MR-neuronavigated tFUS delivered to the human anterior insula and frontal operculum on target - locked EEG event related potentials}

Comparing across aIns/fO tFUS and Sham groups using permutation statistics revealed that event related potentials (ERPs) show an earlier onset, and larger distractor-elicited frontocentral negative (D-N1, $98 \pm 2 \mathrm{~ms}$ ) in all emotion and congruency conditions (Figure 2A). Significant differences between groups begin as early as $28 \mathrm{~ms}$ and last up to $112 \mathrm{~ms}$ in the neutral congruent condition at FCz. Comparison of scalp potentials indicates this difference is significant in frontal and right parietal electrodes (see Figure S1 for ERPs at Fz and P4). No differences across groups at the first distractor-elicited positive peak (D-P1, $146 \pm 2 \mathrm{~ms}$ ) were found. At the first target-elicited negative peak (T-N1, $229 \pm 5 \mathrm{~ms}$ ), the tFUS group exhibited a significantly greater amplitude peak than the Sham group in both frontal and parietal electrodes. This effect is significant at $\mathrm{FCz}$ in the neutral congruent and incongruent conditions, as well as the fear congruent condition. No significant differences were found at peaks P2 $(341 \pm 3 \mathrm{~ms})$ or $\mathrm{N} 2(394 \pm 4 \mathrm{~ms})$ at $\mathrm{FCz}$, however $\mathrm{N} 2$ is significantly larger in the tFUS group at frontal and right parietal electrodes (Figure S1), although no difference in incongruent - congruent potential was observed.

The P3 peak ( $479 \pm 4 \mathrm{~ms}$ ) showed an earlier onset and larger amplitude in the tFUS group compared with Sham in both congruent conditions. Additionally, in both the neutral and fear incongruent conditions, the tFUS group exhibited a more positive late potential (LP, approximately $520-630 \mathrm{~ms}$ ) than the Sham group following the initial P3 peak. A similar patter in early response activation, T-N1, and N2/P3 is also seen across groups in oddball trials (Figure S2).

Isolating individual subjects' peak-to-peak amplitudes at FCz confirmed this result (Table S2). In all conditions D-N1 is greater amplitude in the tFUS group (fear congruent: $p=0.025$, neutral congruent: $p=0.003$, fear incongruent: $p=0.001$, neutral incongruent: $p=0.009$ ). In the fear and neutral congruent, as well as neutral incongruent conditions, T-N1 - P2 peakto-peak amplitude is significantly larger in the tFUS (fear congruent: $5.33 \pm 0.42 \mu \mathrm{V}$, neutral congruent $4.83 \pm 0.68 \mu \mathrm{V}$, neutral incongruent $5.67 \pm 0.58 \mu \mathrm{V}$; median $\pm \mathrm{SEM}$ ) than the Sham group $(3.39 \pm 0.45 \mu \mathrm{V}, 4.83 \pm 0.68 \mu \mathrm{V}, 4.23 \pm 0.51 \mu \mathrm{V}),(p=0.007, p$ $=0.047, p=0.023)$. Additionally, in both the fear $(p=0.007)$ and neutral $(p=0.003)$ congruent conditions, the N2 - P2 amplitude was larger in the tFUS group $(1.92 \pm 0.25 \mu \mathrm{V}, 2.47 \pm 0.27 \mu \mathrm{V})$ than Sham $(0.68 \pm 0.39 \mu \mathrm{V}, 0.20 \pm 0.27 \mu \mathrm{V})$. 

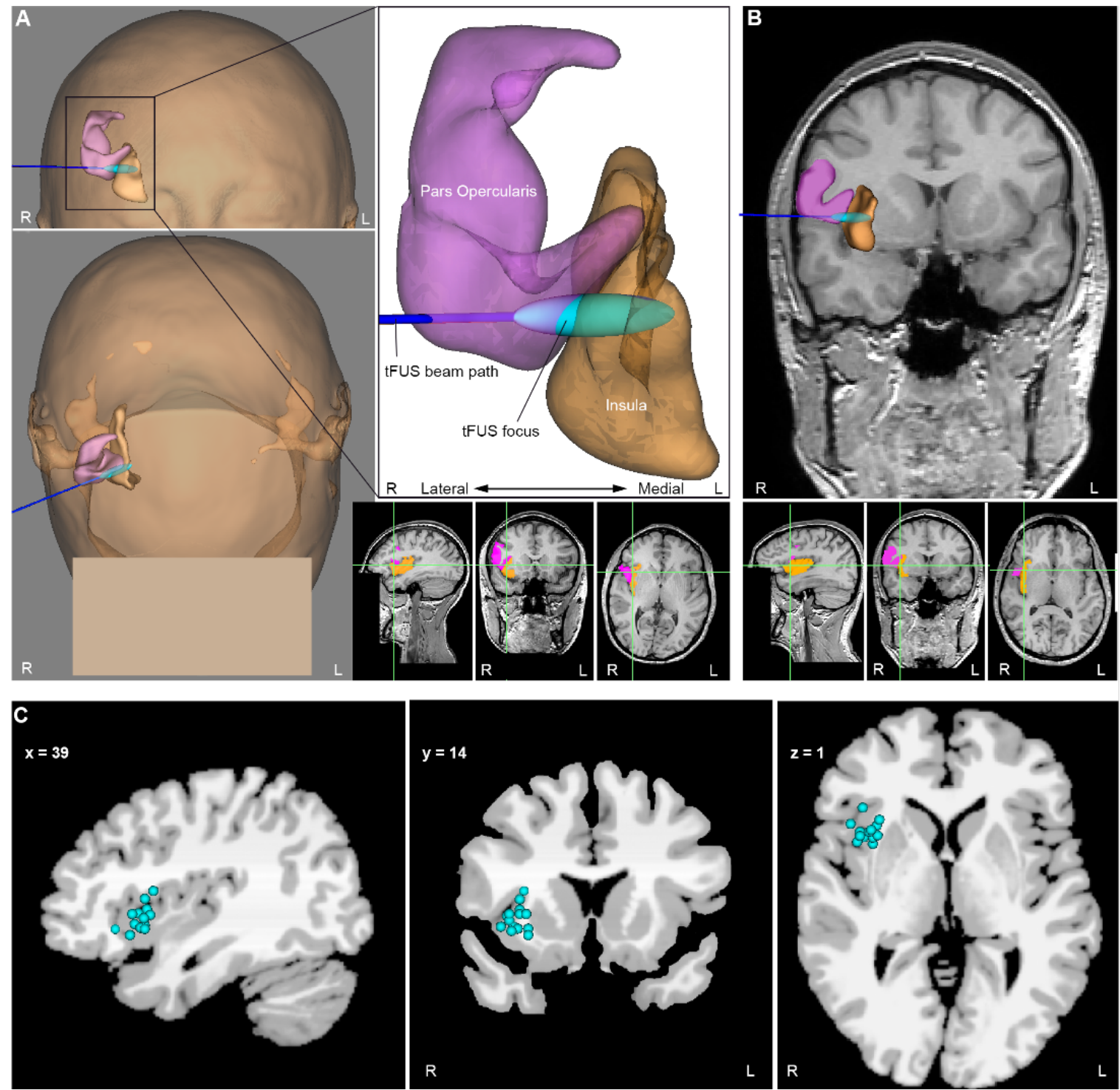

Figure 1. MR-neuronavigated tFUS targeting overlapping regions of the right anterior insula and frontal operculum. (A) 3-D reconstruction of the pars opercularis (pink) and the insula (orange) in a single subject. The tFUS beam path (blue) and focus (cyan ellipsoid) are plotted. Left panel displays 3-D reconstruction of the front and top view of the subject's head; once the tFUS transducer and the subject were registered to the MRI, this representation helped guide proper placement of the transducer. Lower right panel displays the tFUS focal point on the sagittal, coronal, and transvers MRI slices; the gray matter of the pars opercularis and insula are highlighted. (B) A second subject, upper panel displays 3-D reconstruction of pars opercularis and insula overlaid on the coronal MRI. As in A, stimulation focus overlaps the gray matter of the pars opercularis and anterior insula. (C) Stimulation location foci of all subjects overlaid on average brain. Letters at the bottom of the image designate right (R) and left (L). Slices displayed at MNI coordinates $(x=39, y=14, z=1)$. Related to Table S1.

No differences were found across groups in peak latency except at N2 in the neutral congruent condition (Table S3). N2 peak latency was earlier in the tFUS group (374 $\pm 5 \mathrm{~ms}$ ) than Sham $(402 \pm 4 \mathrm{~ms}, p=0.005)$. This is related to the earlier onset of P3 in the tFUS group described above. No other differences in latency were found (all $p>0.10$ ).

\section{Influence of aIns/fO tFUS on incongruent - congruent difference potentials}

Subtracting congruent from incongruent ERPs produced an incon - con difference potential; each face type was compared across groups using permutation testing (Figure 2B). In the fear condition, there was a significant difference across groups in the time range of T-N1, with congruent trials showing a greater amplitude negative peak than incongruent trials in the Sham group, and visa versa in the tFUS group; both groups exhibit a significant main effect of congruency (RM-ANOVA) at this time. Only the Sham group showed a significant main effect of congruency in the time range of P2, but no group differences were found. Similarly, there was a main effect of congruency in both groups in the time range of N2 and P3, but no 
differences are seen across groups. However in the time range of the LP, there was a significant difference across groups in both the fear and neutral conditions. In the tFUS group LP is more positive in incongruent than congruent conditions, while the Sham group exhibited the opposite effect. This difference is significant for a longer time period in the tFUS group.

Testing peak-to-peak amplitudes using nonparametric Friedman's test within subjects revealed that both groups exhibited a difference between conditions at P2 - N2 (Sham: $\chi^{2}(3)=30.43, p<0.001$, tFUS: $\chi^{2}(3)=22.29, p<0.001$ ) and at N2 P3 (Sham: $\chi^{2}(3)=31.89, p<0.001$, tFUS: $\chi^{2}(3)=19.11, p<0.001$ ) resulting from differences between congruent and incongruent trials (see Table S4 for all post-hoc statistics).

\section{Influence of aIns/fO tFUS on fear - neutral difference potentials}

To access the effect of face emotion on ERPs neutral were subtracted from fear responses to produce a neutral - fear difference potential (Figure 2C). As with the congruency conditions, each condition (congruent, incongruent) was compared across groups. Both groups exhibited a larger D-P1 peak for fear than neutral trials (significant main effect of emotion, RMANOVA). This difference is of larger magnitude and duration in the tFUS group than the Sham group in both congruency conditions. At N2 there was a significant difference across groups in the congruent condition; the Sham group exhibited a slightly more negative potential in the fear than neutral condition, although no significant main effect of emotion was found in this time frame.

Comparing peak-to-peak amplitude from individual subjects within each group using Friedman's nonparametric tests further supports this finding (Table S4). There was a significant difference across conditions in D-N1 - D-P1 amplitude in the tFUS $\left(\chi^{2}(3)=10.54, p=0.014\right)$, but not the Sham group tFUS $\left(\chi^{2}(3)=7.63, p=0.054\right)$. Post-hoc analysis using Wilcox signedranks tests showed that fear incongruent $(\mathrm{Mdn}=7.22 \mu \mathrm{V})$ was significantly larger than neutral incongruent $(\mathrm{Mdn}=6.67 \mu \mathrm{V}, p$ $=0.032)$, and neutral congruent $(\mathrm{Mdn}=6.25 \mu \mathrm{V}, p=0.032)$. 

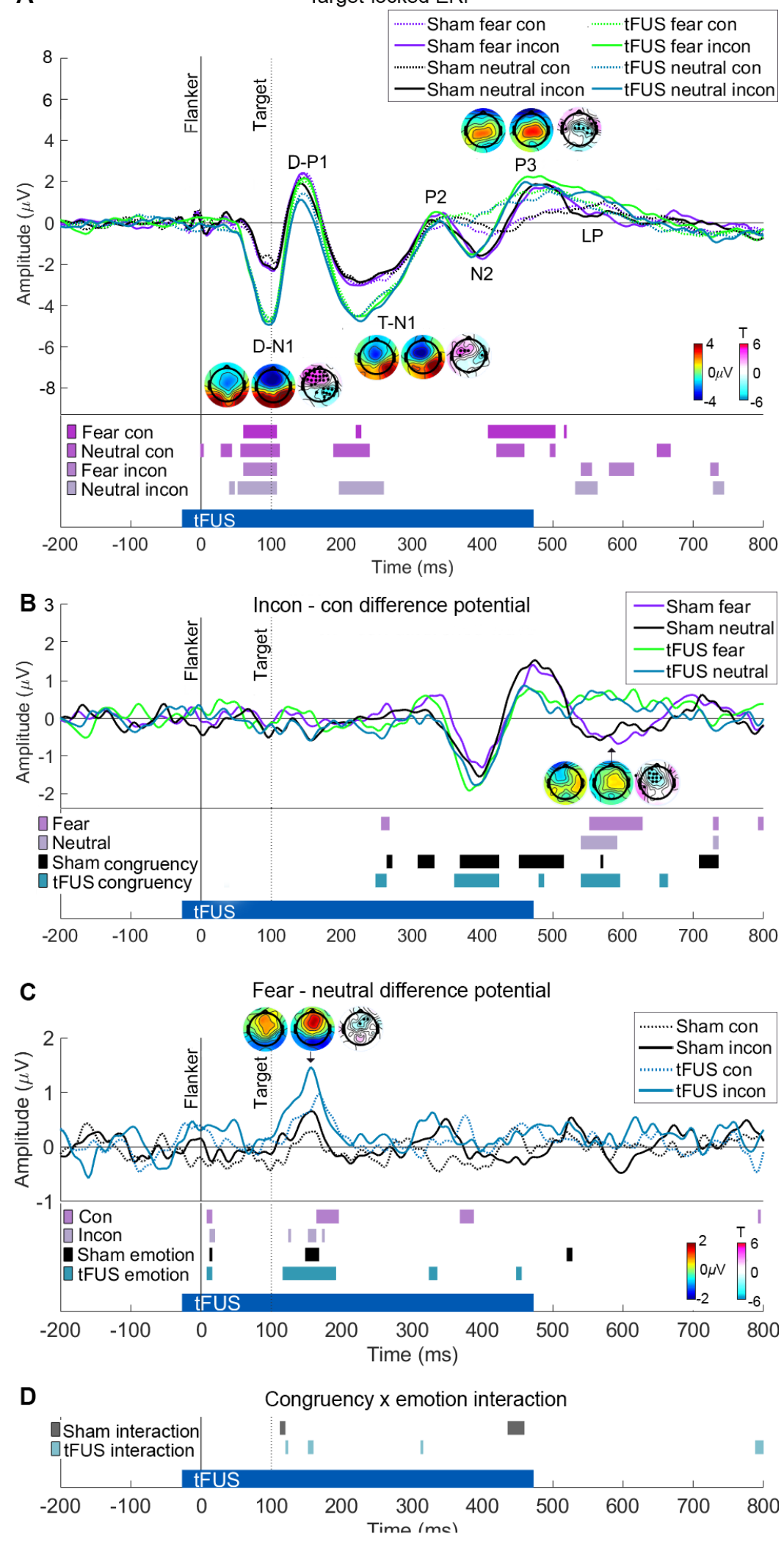

Figure 2. Target-locked ERPs at FCz compared across groups, two congruency and two emotional distractor conditions. (A) ERPs at FCz for each group and all conditions, ERP peaks labeled. Distractor arrows and faces appeared at 0 ms (flanker) and target arrow appeared at $100 \mathrm{~ms}$ (target). Scalp potential maps are displayed for the fear congruent condition where there are significant differences across group (left: Sham, middle: tFUS, right: map of T values, significant electrodes marked with a dot). Scale displayed at right of figure. Time points displayed are: D-N1 (96-104 ms), T-N1 (220 - $228 \mathrm{~ms})$, and P3 (464 - $472 \mathrm{~ms})$. Significant differences across groups for each condition are 
displayed in the lower panel (permutation testing, $p<0.05$ ). tFUS stimulation period marked with blue bar at the bottom of the panel (tFUS). (B) Subtraction of congruent from incongruent potential to make incon - con difference potential. Lower panel displays significant differences across groups in incon - con potential for each emotion condition (fear, neutral), as well as significant congruency effects for each group (RM-ANVOA, performed on data in A). Scalp map: fear (580 - $596 \mathrm{~ms}$ ). (C) Subtraction of neutral from fear potential to make fear - neutral difference potential. Lower panel displays significant differences across groups for difference potential in each congruency condition (con, incon), as well as significant main effect of emotion for each group. Scalp maps displayed for D-P1 (152 ms) in the incongruent condition. (D) Congruency $\times$ emotion interaction effect. Abbreviations: con: congruent, incon: incongruent. Related to Figure S1, Table S2, Table S3, Table S4. See also Figure S2.

\section{Influence of aIns/fO tFUS on congruency and emotion ERP interactions}

To further access the affect emotion may have on congruency processing, emotion $\times$ congruency interaction effect was accessed for each group (Figure 2D). Significant interaction effects where seen for both groups in the time between D-N1 and D-P1. Yet in the time range between N2 and P3 a significant effect was seen only the Sham group. Comparing peak amplitudes from individual subjects with Friedman's nonparametric test supports this finding (Table S4).

\section{Influence of aIns/fO tFUS on frontocentral EEG event-related spectral perturbations}

Comparing event-related spectral perturbation (ERSP) data at FCz across groups with RM-ANOVA yields a main effect of group in the delta, theta, and beta bands (Figure 3). In the delta range (1.5 - 3.5 Hz, Figure 3C), it is clear the tFUS group exhibited a larger power response. Permutation testing showed significant differences across groups in the neutral congruent, and fear congruent, and fear incongruent condition. In the theta range $(4-8 \mathrm{~Hz})$, the event-locked theta peak is of shorter duration in tFUS than Sham. Additionally, in the tFUS group there was a small theta suppression following the initial peak that is not seen in the Sham group. Both the Sham and tFUS group exhibited a congruency effect during this time frame (higher power in incongruent than congruent trials), with no differences across groups. In the alpha range $(8-11 \mathrm{~Hz})$ there was no difference across groups in power. Both groups exhibit a main effect of congruency (Figure 3D), with congruent trials having a larger and earlier theta suppression than incongruent trials. On neutral trials this effect is larger in the tFUS group than Sham as the tFUS group showed greater alpha suppression. Additionally, significance between incongruent and congruent alpha suppression lasts longer in the Sham than tFUS group. Examining the incon - con contrast power during this time frame, there was a significant difference across groups in the neutral condition, and a significant congruency $\times$ emotion interaction effect in the tFUS group. There was a greater alpha rebound following the initial suppression in congruent than incongruent trials in both emotional conditions in the Sham group, but in the tFUS group this is only the case for the fear condition, and the opposite is true for neutral trials.

In the low beta range $(12-22 \mathrm{~Hz})$ the tFUS groups exhibited more event-locked beta suppression than Sham (main effect of group, RM-ANOVA). Permutation statistics confirm there was a significant difference across groups in the neutral and fear congruent conditions, and fear incongruent condition. Additionally, there was higher post-suppression beta activation $(\sim 1000 \mathrm{~ms})$ in the tFUS group than the Sham group. But groups exhibit more suppression in the incongruent than congruent trials and a significant main effect of congruency. However this effect is greater in the tFUS group than Sham group. Comparing incon - con contrast power across groups, there was a main effect of group, and a significant difference across groups in the fear condition using permutation testing.

In the high beta range $(22-40 \mathrm{~Hz})$, similar to the lower beta, there was more event-locked beta suppression in the tFUS group than sham with significant differences across groups in the fear incongruent condition. Comparing incon - con contrast, but groups exhibited some significant effect of congruency, but there are no differences across groups. 
A
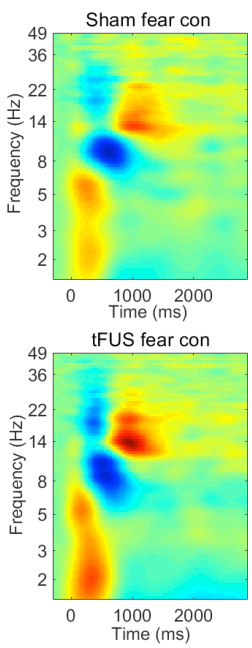

C

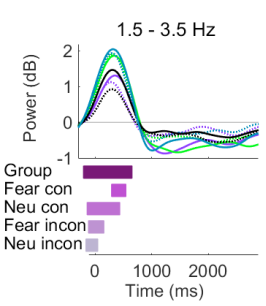

D Incon - con
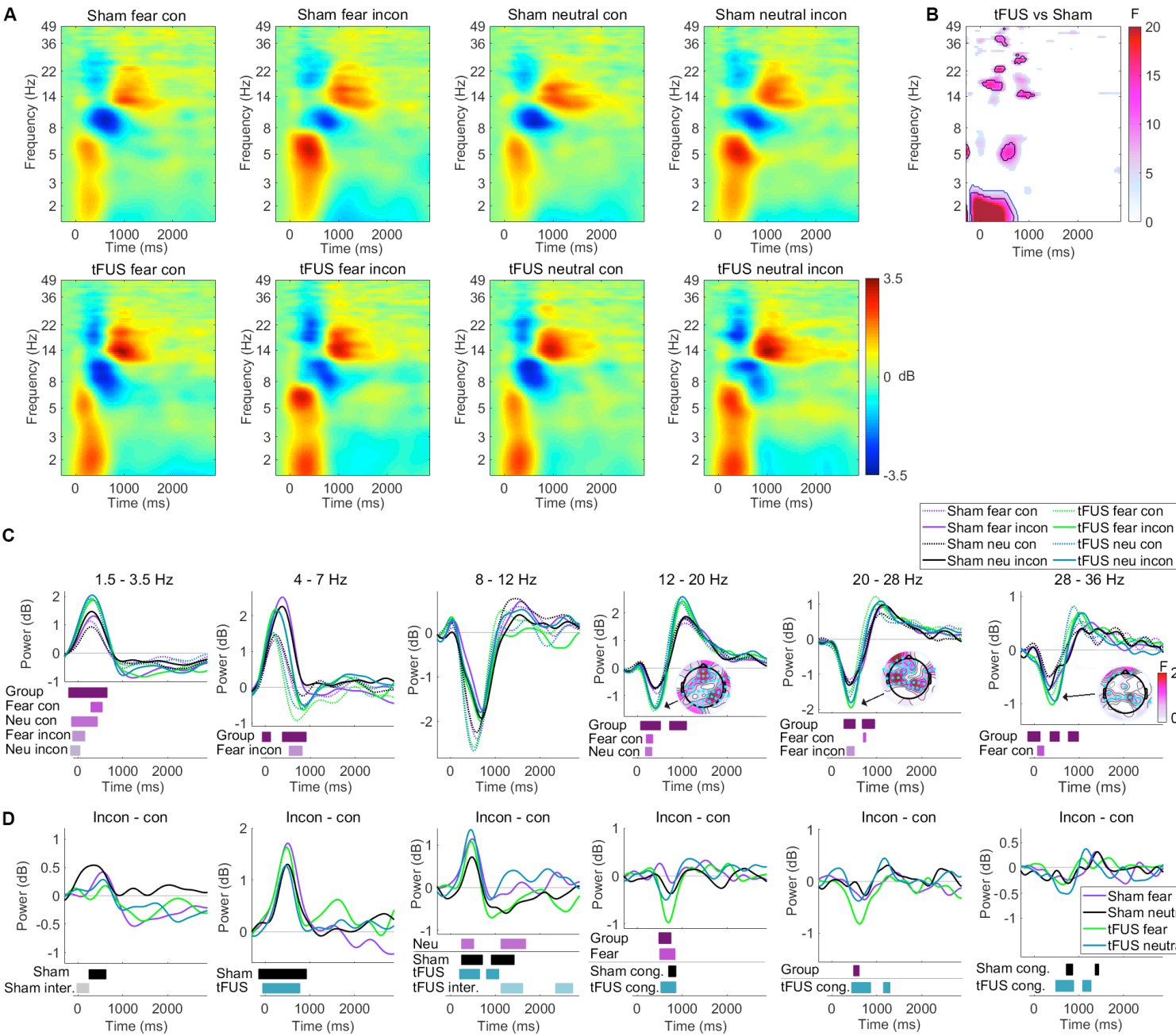

B
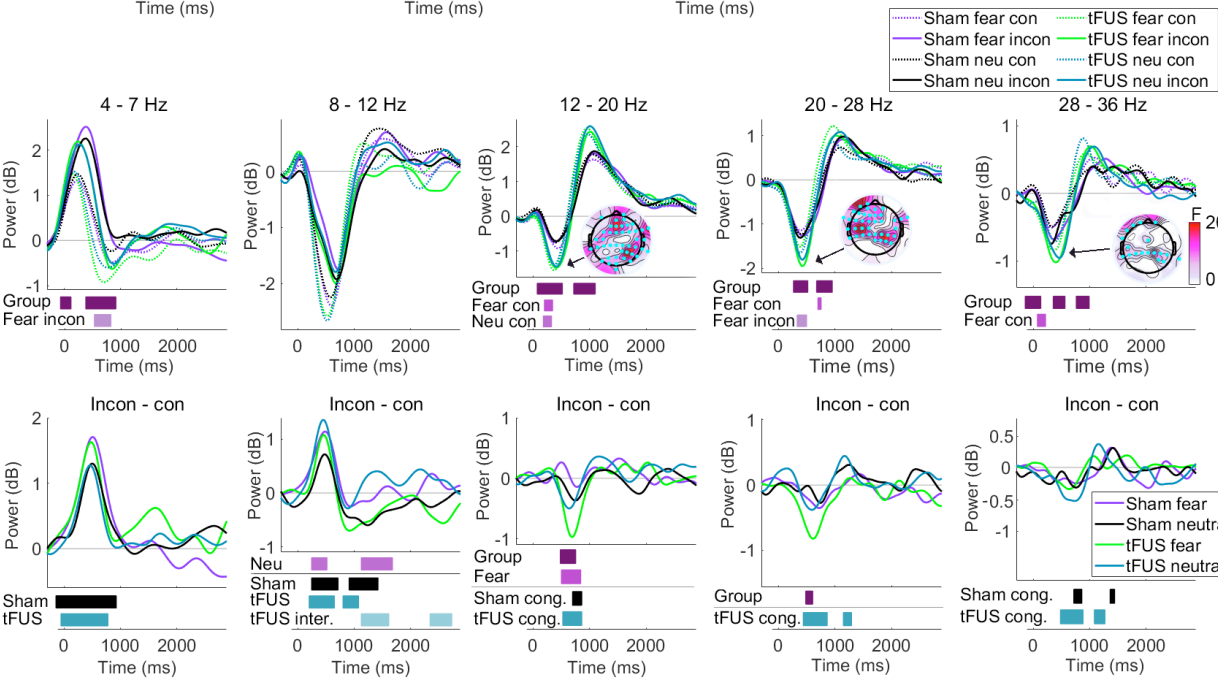

.......tFUS neu con

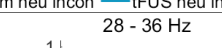

Figure 3. Frontocentral event-related spectral perturbation data. (A) ERSP data at FCz for each trial condition (dB power over baseline). (B) Significant main effect of group (F-values, RM-ANOVA, only $p<0.05$ shown; blue outline: cluster-based threshold outlined; black outline: FDR correction. (C) Power over time in various frequency bands. Lower panel displays significant main effect of group (RM-ANOVA), as well as significant differences in each condition (permutation testing). Scalp maps display significant main effect of group at peak beta suppression (12-20 Hz, 40-360 ms; 20-28 Hz, 100-500 ms; 28-36 Hz, 240-500 ms. Electrodes significant after FDR correction marked with cyan dot. (D) Subtraction of congruent from incongruent power to create incon-con contrast plots for each frequency band in C. Lower panel displays significant differences across groups, as well as main effect of congruency for each group (cong., RM-ANVOA), and congruency $\times$ emotion interaction (interaction). Related to Figure S3 Figure S3 and Figure S4.

Comparing across groups with RM-ANVOA at frontal electrodes (pooled AF3, AFz, and AF4), it is clear that the tFUS group exhibited both an earlier onset and larger magnitude initial event-locked beta suppression and subsequent beta activation (Figure 4). Permutation testing performed across groups in the beta band $(12-20 \mathrm{~Hz})$ confirms that there were significant differences across groups at the initial beta suppression in the fear congruent, neutral incongruent, and fear incongruent. At the beta peak activation (peak 1000 ms) there are significant differences across groups in the fear and neutral congruent, as well as neutral incongruent condition. 

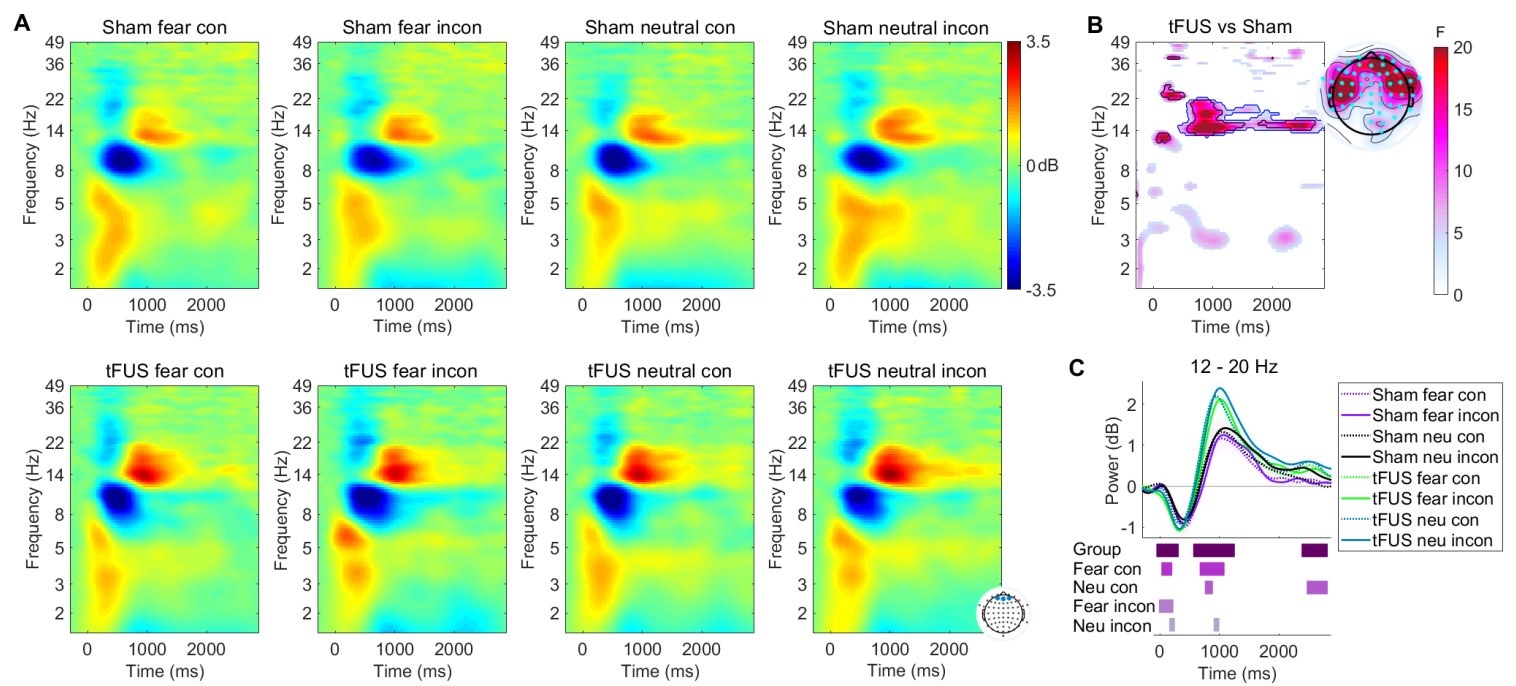

Figure 4. Earlier and larger beta response in tFUS Frontal time-frequency data. (A) ERSP data at frontal electrodes (AF3, AFz, AF4) for each condition (Sham top row, tFUS bottom row). (B) Significant main effect of group (RM-ANOVA, $\mathrm{p}<0.05$ ). Cluster-based threshold outlined in blue, FDR correction in black. Scalp map to the right plots the significance across groups in the low beta range $(12-20 \mathrm{~Hz}, 800-$ $1000 \mathrm{~ms}$ ); electrodes significant after FDR correction marked with a cyan dot. (C) Power over time in the low beta band (12-20 Hz). Significant differences across groups for each condition, as well as the main effect of (Group, RM-ANOVA), are displayed in lower panel.

\section{Influence of aIns/fO tFUS on error-related potentials}

Response-locked ERP data was compared across groups with permutation testing. On error trials, the tFUS group exhibited a larger error-related negativity (40 ms, ERN) at frontal electrodes (Figure 5A inset), and the error - correct potential at frontocentral electrodes (Figure 5B). Comparing each time point of error-locked response at $\mathrm{FCz}$, there was a faster recovery from the ERN peak to the following positive peak in the tFUS group compared with Sham group (Figure 5A). There was also a difference across groups in error ( -400 - $-50 \mathrm{~ms}$ before response) and correct responses $600-300 \mathrm{~ms}$ prior to and at the time of response. 
A

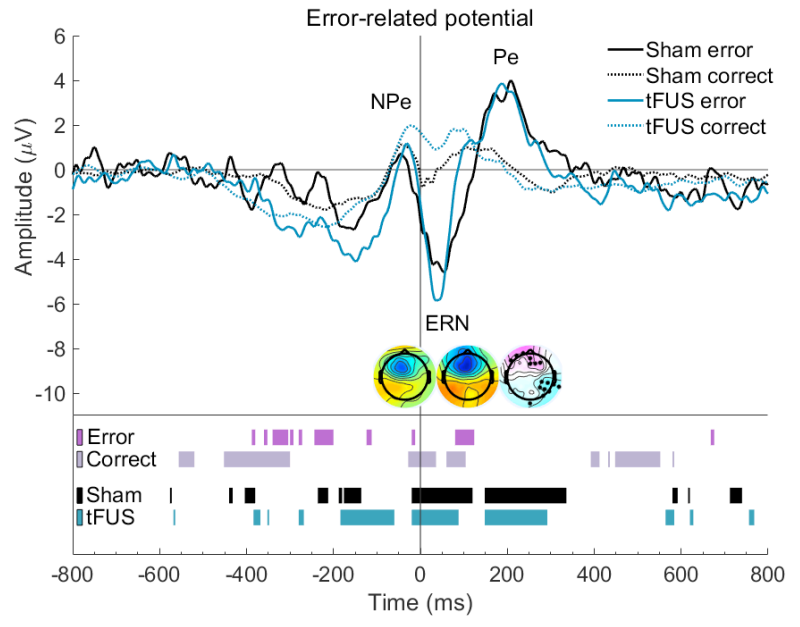

C

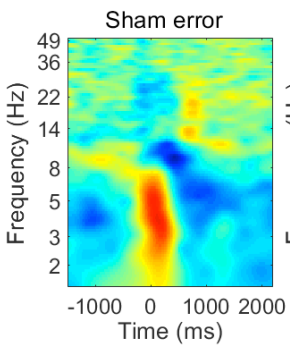

tFUS error

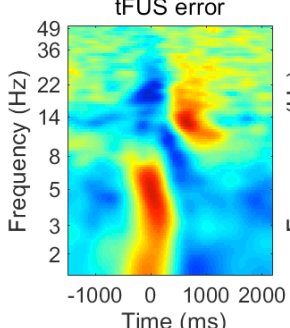

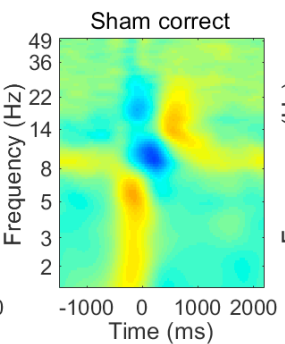

tFUS correct

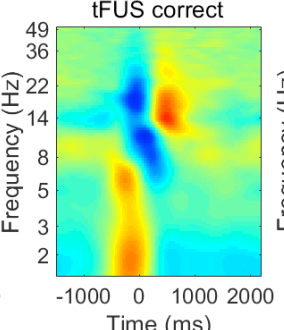

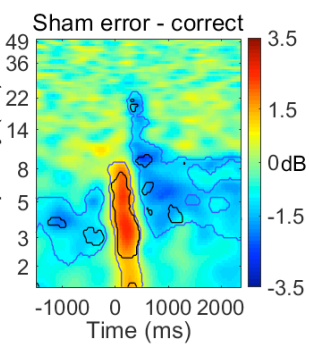

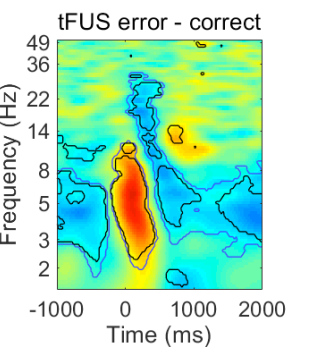

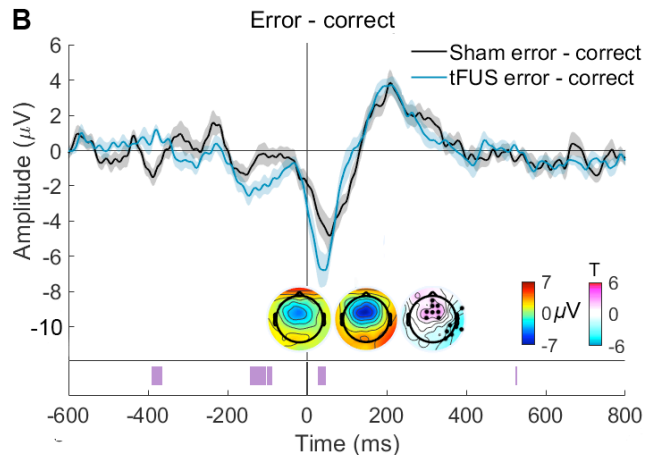

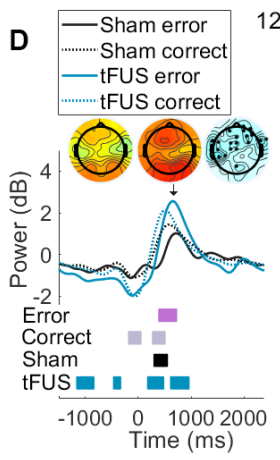

$12-16 \mathrm{~Hz}$
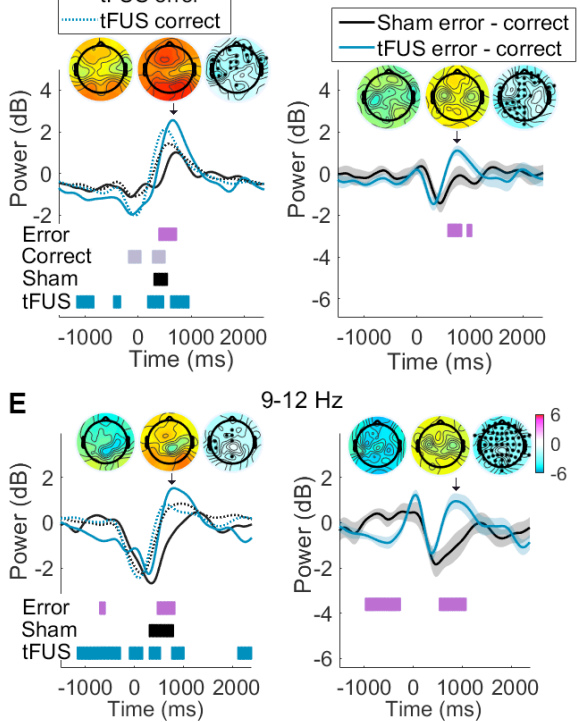

9-12 Hz

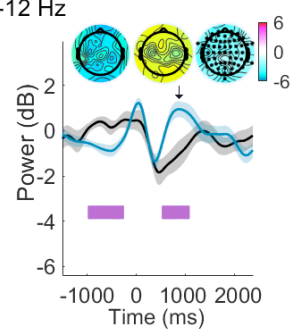

Figure 5. Error Response. (A) Response-locked error related potentials for correct and erroneous responses (response, 0 ms) at FCz. Scalp potentials for ERN peak displayed (40 ms; left: Sham, middle: tFUS, right: map of T values from permutation testing with significant electrodes labeled with a black dot, scales displayed in B. Lower panel displays significant differences across groups (error, correct), as well as error vs. correct within groups (Sham, tFUS) (permutation testing $p<0.05$ ). (B) Subtraction of correct from erroneous response-locked potentials in A. Scalp maps displayed error - correct ERN negative peak at $40 \mathrm{~ms}$ as in A. (C) ERSP for error (left), correct (middle), and error - correct contrast (right) (dB power over baseline). On error - correct plots, significant differences between correct and error responses are outlined (blue: $p<0.05$ cluster-based threshold, black: FDR correction). (D) Power in the low-beta band (12 - $16 \mathrm{~Hz}$ ) for error and correct trials (left, statistics displayed below as in A and error - correct contrast power (right, statics represent permutation testing across groups for error-correct contrast, $p<0.05$ ). Scalp maps of post-error low-beta peak power displayed above plot correspond to time point indicated by the arrow, power scale same as that in B for Sham and tFUS scalp maps, scale for t-map (right-most scalp map) displayed in D. (E) Power in the alpha band $(12-16 \mathrm{~Hz})$ for error and correct trials (left) and error - correct contrast power (right). Statistics displayed as in C.

\section{Influence of aIns/fO tFUS on error-related spectral perturbations}

Comparing ERSP data across groups at FCz for correct and error responses, both groups exhibit a much larger theta response for error than correct trials (Figure 5B), yet only the tFUS group exhibited a larger response for error than correct trials in the alpha and low beta range (Figure 5B, 5C and 5D). Comparing error - correct contrast power (Figure 5C and 5D), the tFUS group exhibited differences with the Sham group post-response across the whole scalp in the alpha range ( 9 - 12 Hz), and at frontal, left parietal, and midline electrodes in the low-beta range (12-16 Hz). Examining error and correct responses, it is clear that both groups exhibit a suppression of alpha at the time of response, followed by an alpha recovery (Figure 5D). While there was no difference across groups on correct responses, the tFUS group exhibited significantly higher reactivation of alpha than Sham in frontocentral electrodes. During the post-error recovery of alpha power, the tFUS group showed significantly higher power in error than correct trials, and at the post-recovery peak, higher power than baseline. Additionally, prior to an erroneous response, the Sham group does not show any differences in alpha power between error and correct 
trials, but the tFUS group showed significantly less alpha activity on trials where errors were committed, and significantly higher alpha activity at the time of error compared to correct trials $(0 \mathrm{~ms})$. There was also a significant group difference in error-correct contrast power beginning 1000 ms prior to response.

In the low-beta range, both groups exhibit and post-response beta activation, yet in front-central electrodes, this activation is significantly greater in the tFUS group than Sham on both correct and error responses (Figure 5C). Additionally, the tFUS group exhibited significantly higher low-beta activation on error than correct response, and visa-versa in the Sham group; with a significant difference across groups in error-correct contrast power at this time. The tFUS group also exhibited significantly lower low-beta activity on error compared with correct trials prior to responding, while no differences were observed in the Sham group.

\section{Influence of aIns/fO tFUS on heart rate and heart rate variability}

To access the effect of emotional face distractors on physiologic response in the case of Sham vs. tFUS stimulation to the aIns/fO, heart-rate metrics were recorded during the final three minutes of the baseline flanker trials (no faces, both groups received sham stimulation), and first three minutes of main trials (faces presented as distractors behind flanker task, and tFUS group received active stimulation on each trial, while the Sham group received sham). Several metrics of heart rate variability (HRV) were calculated and compared (see Methods). Data was analyzed with two-factor, mixed-measures RMANOVA ([2 groups (sham, tFUS) $\times 2$ time points (baseline, faces with stimulation)] (Figure 6, Table S5).

The results showed a significant group $\times$ time interaction in the standard deviation of the normal-to-normal heartbeat $(\mathrm{SDNN})\left[\mathrm{F}_{\mathrm{SDNN}}(1,25)=5.86, p=0.023, \eta_{\mathrm{p}}{ }^{2}=0.19\right]$ and SD2 (long-term HRV, see Methods) $\left[\mathrm{F}_{\mathrm{SD} 2}(1,25)=5.87, p=0.023, \eta_{\mathrm{p}}{ }^{2}=\right.$ 0.19]. Post-hoc statics show that in the SDNN, the Sham group showed a significant decrease $(-20 \pm 4 \%$, mean \pm SEM) from baseline to onset of face distractors $(p=0.001)$, while no significant change was seen in the tFUS group $(-2 \pm 6 \%, p=0.67)$. Similarly, the Sham group showed a significant decrease in SD2 at the onset of faces $(-22 \pm 4 \%, p=0.001)$, while the tFUS group does not $(-2 \pm 7 \%, p=0.71)$. No other significant main effects of group, faces or interaction effects were found for heart rate (HR), mean beat-to-beat interval (RR), SD1 (short-term HRV), or SD1/SD2 ratio (F $\leq 3.42, p \geq 0.076$, Figure 6, Table S5). Unpaired t-test showed no significant differences at baseline (all $T \leq 1.62, p \geq 0.067$ ).
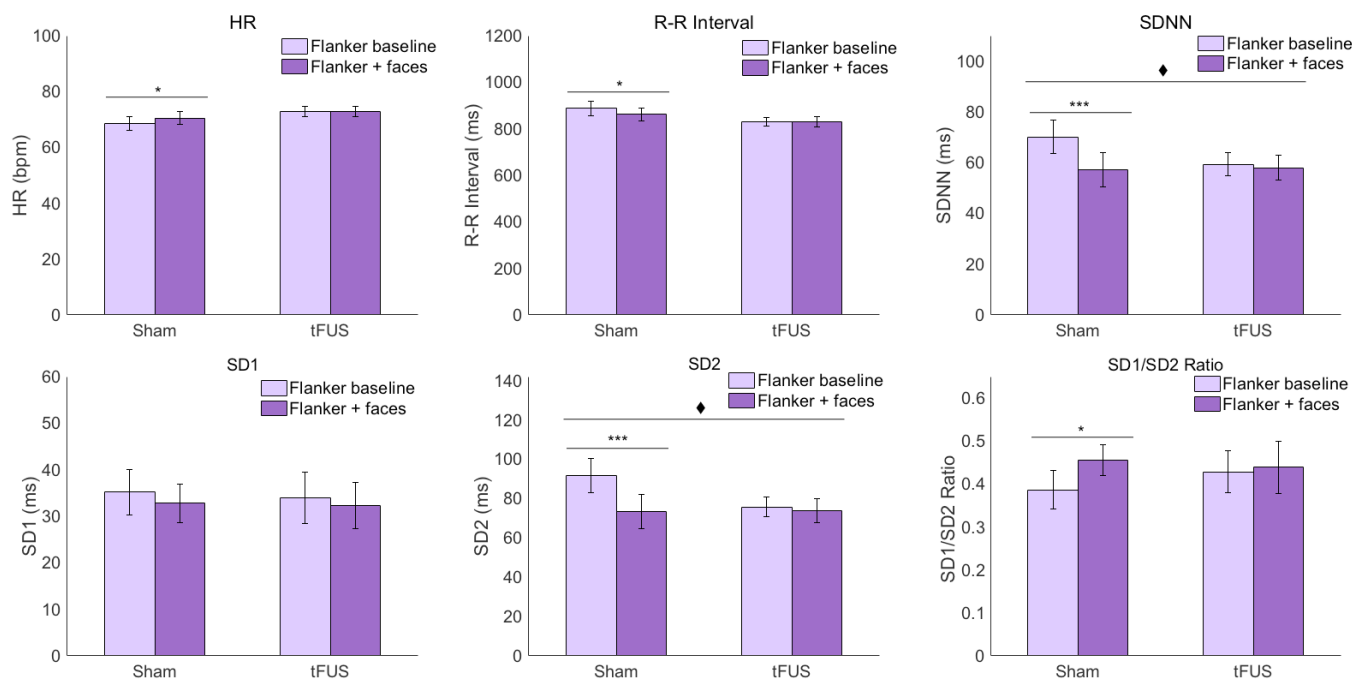

Figure 6. Heart rate and heart rate variability metrics. Comparison of HRV metrics collected in the final three minutes of subjects performing the baseline flanker trials (Baseline: simple flanker, no faces, both groups received sham) and first three minutes of the main trials (Flanker + faces: faces presented as distractors behind flanker arrows, tFUS group received active situation on each trial). Displayed statics represent the results of RM-ANOVA ( indicates significant group $\times$ time interaction, $p<0.05$; post-hoc tests Bonferroni-corrected: * $p$ $\left.<0.05,{ }^{* *} p<0.01,{ }^{* * *} p<0.001\right)$. Related to Table S5. 
Table 1. Baseline-subtracted reaction time does not differ across groups.

\begin{tabular}{|c|c|c|c|c|c|}
\hline \multicolumn{3}{|c|}{ Baseline-subtracted RT (ms) } & \multicolumn{3}{|c|}{ Mann-Whitney } \\
\hline Congruent & Sham & tFUS & $p$ & $\mathbf{U}$ & $\mathbf{W}$ \\
\hline Fear & $31 \pm 7$ & $19 \pm 7$ & 0.57 & 22.0 & 32.0 \\
\hline Neutral & $27 \pm 7$ & $21 \pm 6$ & 0.51 & 21.0 & 31.0 \\
\hline Oddball & $39 \pm 7$ & $40 \pm 8$ & 0.97 & 27.0 & 37.0 \\
\hline Post & $-6 \pm 9$ & $-7 \pm 7$ & 0.65 & 33.0 & 43.0 \\
\hline \multicolumn{6}{|c|}{ Incongruent } \\
\hline Fear & $7 \pm 6$ & $5 \pm 6$ & 0.57 & 22.0 & 32.0 \\
\hline Neutral & $13 \pm 6$ & $-3 \pm 6$ & 0.51 & 21.0 & 31.0 \\
\hline Oddball & $17 \pm 13$ & $9 \pm 8$ & 0.97 & 27.0 & 37.0 \\
\hline Post & $-3 \pm 6$ & $-1 \pm 5$ & 0.65 & 33.0 & 43.0 \\
\hline
\end{tabular}

Group-level statics are displayed on the right (Mann-Whitney U test). RTs displayed as median \pm SEM (ms). Related to Figure S2, see also Figure S3 and Table S6.

\section{Influence of aIns/fO tFUS on baseline-subtracted reaction times}

Mann-Whitney tests indicated no significant differences across groups in baseline-subtracted reaction time (RT) in any trial condition (all $\mathrm{U} \geq 21.0, p \geq 0.51$ (Table 1). Although there was no difference across groups in baseline-subtracted RT, there were differences in within group comparison. Friedman's test showed that in the Sham group, there was a significant difference between RT's in the baseline, fear, neutral, oddball, and post-experiment trials in the congruent condition $\left[\chi^{2}(4)=\right.$ 28.95, $p<0.001$ ] (Figure 7). Post-hoc analysis using Wilcox signed-rank tests showed that for congruent trials, oddball trials $(\mathrm{Mdn}=353 \mathrm{~ms})$ were significantly slower than baseline trials $(\mathrm{Mdn}=334 \mathrm{~ms}, p=0.002)$, and post-stimulation trials $(\mathrm{Mdn}=$ $306 \mathrm{~ms}, p<0.001)$. In the tFUS group, there was also a significant difference between baseline, fear, neutral, oddball, and poststimulation trials in congruent condition $\left[\chi^{2}(4)=22.50, p<0.001\right]$. Post-hoc tests show that in the congruent condition, fear (Mdn $=326 \mathrm{~ms}, p=0.049)$ and oddball trials $(\mathrm{Mdn}=332 \mathrm{~ms}, p=0.007)$ were significantly slower than baseline trials (Mdn $=$ $305 \mathrm{~ms}$ ) and post-experiment trials (310 ms; fear: $p=0.015$, oddball: $p=0.002)$. For incongruent trials, the Sham group exhibited a significant differences between conditions $\left[\chi^{2}(4)=10.94, p=0.027\right.$, all post-hocs $p>0.14$ after Bonferronicorrection], but the tFUS group does not $\left[\chi^{2}(4)=0.91, p=0.82\right]$.
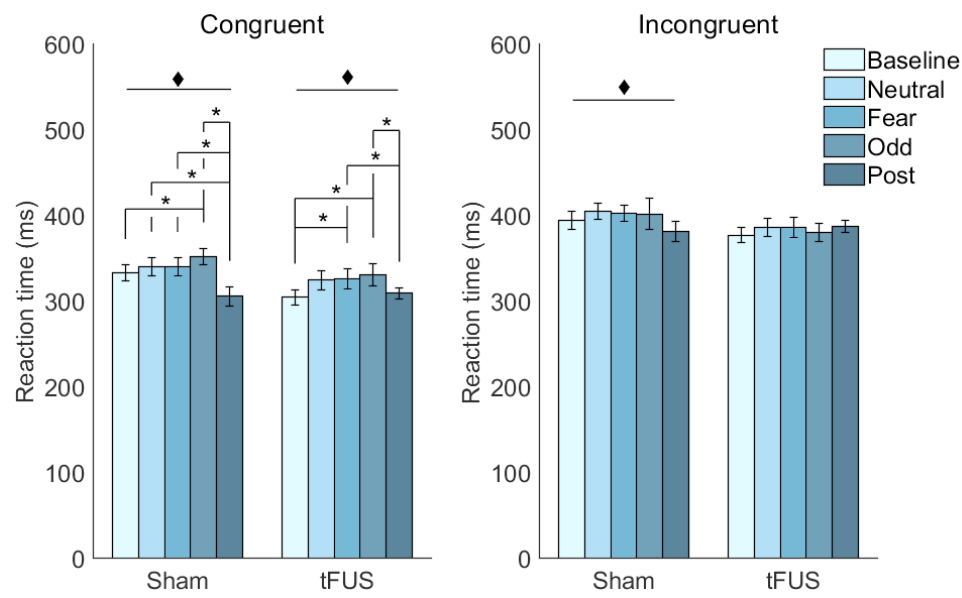

Figure 7. Within-group RT analysis. Median RTs for each trial condition (baseline: simple flanker, no faces, no stimulation), neutral, fear and oddball faces with stimulation, and post-experiment trials: identical to baseline). RTs displayed by group for congruent trials on the left, and incongruent trials on the right. ( indicates significant difference across conditions, Friedman's non-parametric test; $*$ p < 0.05 , Bonferroni-correct post-hoc tests). See also Figure 1, Figure S3. 


\section{Influence of aIns/fO tFUS on conflict adaption}

To assess conflict adaption and its interaction with emotional face distractors and tFUS to the aIns/fO, trials were separated based on congruency of the previous trials (congruent, incongruent). Congruency effect in RT was then calculated by subtracting median congruent from incongruent RT's. There were no differences between groups (Mann-Whitney U test, all $\mathrm{U} \geq 82.0, p \geq 0.48$ ).

Additionally, baseline, neutral, fear, and post-stimulation RT's were compared within group (Figure 8). Similar to RTs, for trials following congruent trials, both the Sham group $\left[\chi^{2}(3)=19.58, p<0.001\right]$ and the tFUS group $\left[\chi^{2}(3)=11.40, p=\right.$ 0.010] exhibited a significant difference across conditions. Post-hoc tests show that in the Sham group, neutral congruency effect (Mdn $=65 \mathrm{~ms}$ ) was significantly lower than baseline (Mdn $=88 \mathrm{~ms}, p=0.016)$ and post-stimulation trials $(\mathrm{Mdn}=99 \mathrm{~ms}$, $p=0.002)$, while fear (Mdn $=60 \mathrm{~ms}$ ) was significantly lower than post trials $(p=0.010)$. In the tFUS group, fear congruency effect ( $\mathrm{Mdn}=62 \mathrm{~ms}$ ) was significantly less than post ( $\mathrm{Mdn}=80 \mathrm{~ms}, p=0.032)$. However, for trials following incongruent trials, only in the Sham group was there a significant difference between conditions [Sham: $\chi^{2}(3)=13.10, p=0.004 ; \mathrm{tFUS:} \chi^{2}(3)$ $=5.57, p=0.13]$. Post-hoc tests show that in the Sham group, the congruency effect for baseline trials (Mdn $=78)$ was significantly greater than fear trials ( $\mathrm{Mdn}=60 \mathrm{~ms}, p=0.016)$ and neutral trials (Mdn $=61 \mathrm{~ms}, p=0.041$ ).
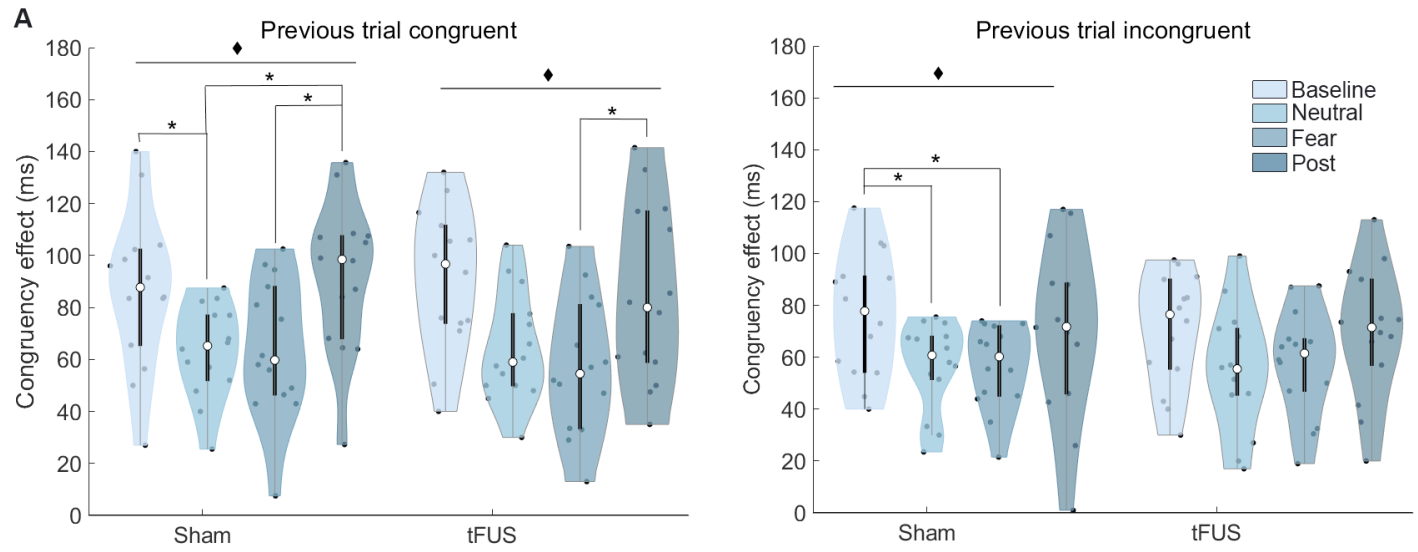

Figure 8. Conflict adaption: congruency effect in reaction time separated by previous trial congruency face trial conditions compared within group. Violin plots display congruency effect by group (median incongruent RT minus congruent RT) for trials in which the previous trial was congruent (left plot) or incongruent (right plot). Trial face types (baseline, neutral, fear, post-stimulation) are compared within group. White dots indicate median, and thick dark-grey bars indicate first and thirds quartiles of the data. ( indicates significant difference across conditions, Friedman's non-parametric test; ${ }^{*} \mathrm{p}<0.05$, Bonferroni-corrected post-hoc tests). See also Figure 7.

\section{Influence of aIns/fO tFUS on response accuracy}

Mann-Whitney tests indicated no significant differences across groups in any trial condition (all $\mathrm{U} \geq 79.0, p \geq 0.33$ (Table S6).

\section{Influence of aIns/fO tFUS on post error slowing}

Mann-Whitney test indicate that in post-error fear congruent trials, RT is significantly slower in the Sham $(\mathrm{Mdn}=390)$ compared with tFUS (Mdn $345 \mathrm{~ms}, \mathrm{U}=38.1, p=0.009$, Figure 8). No other conditions were significant across group (all $p>$ 0.18). This result was supported by within group testing with Wilcox signed-ranks test indicated on post error congruent trials, median-subtracted RT was slower on neutral ( $\mathrm{Mdn}=28 \mathrm{~ms}$ ) than fear (Mdn $=5 \mathrm{~ms}, \mathrm{Z}=86, p=0.035$ ) trials (Figure S9). No other significant within group effects were found $(\mathrm{Z}<36, p>0.18)$.

\section{Influence of aIns/fO tFUS on acute mood}

For positive PANAS scores mixed-measures, two-way RM-ANOVA ([2 groups (sham, tFUS) $\times 2$ time points (baseline, immediately following experiment completion)] indicated a significant main effect of time $\left[F_{\text {panAS }}(1,25)=21.66, p<0.001, \eta_{p^{2}}\right.$ $=0.46$, with positive scores being higher at baseline (mean $=31.0$ ), then immediately following experiment completion (mean = 25.3) (Table S7). Analyzing individual PANAS metrics reveals that this effect is due to interest and motivation, there was a significant decrease in the following categories: 'interested', 'excited', 'strong', 'enthusiastic', and 'attentive', but not in 'proud', 'alert', 'inspired', 'determined', or 'active'. There was no main effect of group $\left(F_{\text {panas }}(1,25)=0.17, p=0.69, \eta_{p}^{2}=0.01\right)$ nor a time $\times$ group interaction effect $\left[F_{\text {PANAS }}(1,25)=0.01, p=0.95, \eta_{p}^{2}=0.00\right]$.

For negative PANAS scores, there was no effect of time $\left[\mathrm{F}_{\mathrm{PANAS}}(1,25)=2.60, \mathrm{p}=0.12, \eta_{\mathrm{p}}{ }^{2}=0.09\right]$ or main effect of group $\left[\operatorname{FPANAS}(1,25)=0.16, \mathrm{p}=0.69, \eta_{\mathrm{p}}^{2}=0.01\right]$, nor was there a significant time $\times$ group interaction effect $\left[\operatorname{FPANAS}_{\operatorname{los}}(1,25)=4.20, p\right.$ $\left.=0.051, \eta_{\mathrm{p}}^{2}=0.14\right]$. 


\section{DISCUSSION}

The aim of this study was to investigate the feasibility of modulating attentional networks by stimulating a single brain area (the right aIns/fO) with tFUS and to assess the effects on conflict and emotional distractor processing. EEG, HRV, behavioral responses, and mood were assessed. It was hypothesized that since the aIns/fO plays a substantial role in executive control and emotional distraction (Kanske et al., 2011), and has broad structural and functional connections to both executive and autonomic functions, modulating the region may have broader network-level effects on face distractor processing. Indeed, a pronounced response in early ERP components was observed, as well as larger delta and beta activity, and modulation of theta. Differences were also seen in the task-locked alpha and beta bands, as well as in error responses and post-error RTs. Additionally, HRV decreased in response to emotional face distractors in Sham; however, this was not the case for subjects who received tFUS to the aIns/fO.

\section{Enhancement of early ERP components and heightened responses to fearful faces}

Early ERP components D-N1 and T-N1 (frontocentral negativity, parietal positivity) are significantly larger at frontocentral and right parietal electrodes in subjects receiving tFUS to the right aIns/fO, compared with subjects receiving sham. Additionally D-N1 has an earlier onset in the tFUS group. Also, D-P1, frontal positivity, was of greater amplitude in fear compared with neutral trials consistent as with previous studies (Carlson and Reinke, 2010; Eimer, 2000; Righart and de Gelder, 2006), an effect that is notably larger in the tFUS group, especially on incongruent flanker trials.

These early ERP components are associated with perception and exogenous attention selection (Carretié, 2014), emotional processing (Smith et al., 2003), and emotional distraction (Bretherton et al., 2017; Neumann et al., 2011), and have larger amplitudes in fear-sensitive individuals in response to emotional fear arousal (Dennis and Chen, 2007). Interestingly, these ERP effects are not simply modulated through dopaminergic attention networks, as they are not affected by methamphetamine (Bensmann et al., 2018).

Fear face processing is thought to be mediated through involvement of the insula, amygdala, anterior cingulate, and visual cortex. The insula is involved in conscious but not unconscious fear perception while the amygdala is active in both (Critchley et al., 2002a). Insula activity is specifically related to anxiety response, is hyper-reactive to fear faces in social anxiety disorder, and shows reduced connectivity to the prefrontal regions involved in cognitive control (Klumpp et al., 2012). Early frontal positive potentials have been shown to decrease in attentional fatigue (Boksem et al., 2005) and increase with mindfulness meditation training (Moore et al., 2012), which is known to involve in the insula (Tang et al., 2015). It may be that a pronounced frontocentral fear potential in this study resulted from tFUS affecting the insula's regulation of medial prefrontal areas.

At later ERP amplitudes, there was an earlier onset of P3 in congruent neutral and fear trials, and a difference in LPs in incongruent trials, perhaps due to effects related to saliency, in which the aIns/fO is involved (Cuthbert et al., 2000).

\section{Time-frequency response showed heightened delta and beta response in aIns/fO tFUS group}

In target-locked ERSP time frequency data, the tFUS group showed larger frontocentral delta activity in all conditions and a theta peak that is shorter in duration than the Sham group in the fear incongruent condition. The tFUS group also showed significantly larger frontocentral and parietal beta suppression as well as larger post-suppression beta activation. This effect is especially pronounced in frontal electrodes. Additionally, the frontocentral theta peak was shorter in duration in the aIns/fO tFUS group in the fear incongruent condition. Yet, no differences in congruency effect (incon - con contrast power) were seen in the peak theta range, however there was a significant differences between groups in the lower beta range, the tFUS group showed a larger and longer beta suppression in the fear incongruent condition.

It has been previously demonstrated that larger central delta is distinct from theta contributions, and associated with larger P3 and LP ERP components (Harper et al., 2014, Gilmore, 2010 \#6905), which is consistent the findings presented in this study. Task-related delta is related to decision-making, saliency, and produced in the nucleus accumbens during reward (Bernat et al., 2012; Grace, 1995; Knyazev, 2007). Intracortical recordings in the human insular cortex demonstrate that beta amplitude is modulated by the likelihood of performance feedback (Billeke et al., 2020), and delta phase modulated beta power encodes feedback valance. Beta functions to commutate feedback information to the medial prefrontal cortex from the insula. Given this, differences in delta and beta could very likely be mediated through modulation of the aIns/fO with tFUS.

On error responses, the tFUS group showed a larger amplitude and shorter duration frontal/ frontocentral ERN. In response-locked time frequency data, the tFUS group showed differences compared to Sham in alpha and low beta but not theta. On error trials the tFUS group exhibited an earlier and larger alpha recovery following the response-locked alpha suppression. Error - correct alpha power in this time range is greater in the tFUS over almost all EEG electrodes. The tFUS groups also exhibited more alpha suppression prior to the error as compared with correct trials. Additionally, there was a larger amplitude frontocentral low beta response in tFUS than Sham on both erroneous and correct responses, but was larger on error trials. There was also a group difference in frontal and parietal electrodes for heightened beta in post error responses in error - correct contrast power. 
The ERN is related to cognitive control, attention, and perhaps experience, but not necessarily valence, as it is higher in obsessive compulsive disorder and state worry (Hajcak et al., 2003), but also in meditators (Teper and Inzlicht, 2013). Stronger error-related suppression of alpha as well as parieto-occipital-frontal synchronization is associated with concentration. Attentional lapses have been shown to result in frontal theta as well as posterior alpha suppression, suggesting differential performance monitoring mechanisms. On errors, alpha in parietal electrodes have been linked to phase synchrony with right fronto-inferior electrodes (van Driel et al., 2012). EEG-fMRI research which links the aIns/fO to error processing (Iannaccone et al., 2015). Additionally, it has been suggested that post error beta in central EEG electrodes can act as a marker for decision making and post error adjustments (Fischer et al., 2018). Consistent with the beta differences described previously in target-locked ERSP data, this presents a compelling case for successful modulation of performance monitoring primarily through modulation of delta and beta, but also error-related alpha.

\section{Heart rate variability does not increase in response to faces in tFUS group}

In addition to electrophysiology, physiological heart rate metrics were also compared across groups. The right insula is known to be involved in cardiac (Abboud et al., 2006) and autonomic (specifically parasympathetic) regulation (de Morree et al., 2016), and has direct neural projections to the amygdala and autonomic regulatory nucleoli of the dorsal medulla (Kapp et al., 1985). HRV provides well-studied metrics of autonomic nervous system activity, known to be sensitive to, anxiety (Gaebler et al., 2013; Thayer et al., 2012), and be involved with distraction by fearful faces (Park et al., 2013; Park et al., 2014). The SDNN (standard deviation of RR intervals) is known to decrease with increased workload (Fallahi et al., 2016). SDNN is influenced by both the sympathetic but largely parasympathetic activity via the rise and fall of the heart rate with slow relaxed breathing (Shaffer et al., 2014). This study showed that in the Sham condition, there was indeed a significant decrease in SDNN with the onset of the face distractors yet no change was seen in the tFUS group. SDNN is known to be lower in patients with right insula stroke (Colivicchi et al., 2004).

Analyzing metrics from the Poincaré plot of R-R intervals ( $R R_{i+1}, R_{i}$; see methods), the SD1 (identical to the RMSSD (Ciccone et al., 2017)) which is vagally modulated and though of as short term HRV(Spangler and McGinley, 2020), showed no changes across groups or time. While as with the SDNN, the SD2 significantly decreased in the Sham group at the onset of the face distractors, but no change was seen in the tFUS group. The SD2 is thought to be a long term HRV moving average, but also influenced by short term HRV. SD2 is correlated with baroreflex sensitivity (Guzik et al., 2005), and animal studies have shown that the insula modulates cardiac baroreflex through parasympathetic modulation (Saleh and Connell, 1998). The above findings suggest that tFUS modulated physiology through right insula, and the physiological suppression of HRV seen in the Sham subjects in response to fearful distractors face is blocked by tFUS to the aIns/fO.

\section{Behavioral metrics}

No differences in baseline-subtracted RT or accuracy were observed across groups. However, the Sham group showed a significant RT slow-down with the addition of the fear and neutral face distractors in both congruent and incongruent condition as expected from the literature (Bretherton et al., 2017), whereas this was only the case in the tFUS group in the congruent condition but no significant slowdown from baseline was seen in the tFUS group on incongruent trials.

Similar to reaction time, congruency effect (incon - con RT) showed a significant reduction in the presence of face distractors compared to baseline and post stimulation trials in both group when following a congruent trials. This result was consistent with previous work that demonstrates RT congruency effect decreases with emotionally salient negative distractors (Meng et al., 2019). However on trials following incongruent trials, this was only true for the Sham group. The tFUS group showed no difference in congruency effect with the addition of the faces in the incongruent condition, suggesting that tFUS to the aIns/fO alters salient emotional distraction.

\section{Post error slowing}

Post error fear congruent trials show a slower RT in the Sham than tFUS group. Additionally, the tFUS group showed significantly less post error slowing on fear than neutral congruent trials, demonstrating modulation of post error task processing.

\section{Conclusions}

The aIns/fO is involved emotional processing, but also executive control through monitoring task performance evaluation (Eckert et al., 2009) and set attentional maintenance (Dosenbach et al., 2008), perhaps through integrating saliency. It has been proposed that the insula functions in performance feedback through enhanced awareness of autonomic responses resulting from error (Klein et al., 2007). This study provides evidence that modulation of the aIns/fO with tFUS reduces parasympathetic fear response seen in HRV, and functionally reduces emotional distraction interference on performance, as well as response slowing after errors on fear trials. Additionally, ERP differences were measured across groups in components associated with saliency, emotion, and congruency. These effects are likely meditated here through modulation of event-locked delta and beta with tFUS. These frequencies are known to be involved in modulation of physiologic 
response though interaction with the amygdala, as well as distractor processes in through insular-medial frontal regulation during feedback. Overall, this study demonstrates that tFUS along with neuronavigation methods can be used to target specific anatomical and functional areas to induce larger network modulation effects on conflict processing, cognitive control, and emotional attention.

\section{MATERIALS AND METHODS}

\section{Human Participants and Informed Consent}

All procedures were performed using protocols approved by the Arizona State University Institutional Review Board. Twenty-eight healthy, right-handed adult volunteers with no previous history of neurologic conditions and no current medication use were recruited for this study. All participants had no hearing or uncorrected visual impairments, migraines, or medication use, as well as no contraindications for MRI. Following screening, human volunteers were provided with a final overview of the study prior to providing informed consent.

Subjects were randomly divided into two groups: one to receive active tFUS to the aIns/fO ( 6 female, 8 male, mean age 24.1) and the other to receive sham situation ( 3 female, 11 male, mean age 22.4). Participants were blinded to the mode of stimulation they received; both groups were told they received active stimulation. One subject was from each group was removed from HRV analysis due to poor recording. One subject in the insula group was removed from mood assessment due to survey incompletion.

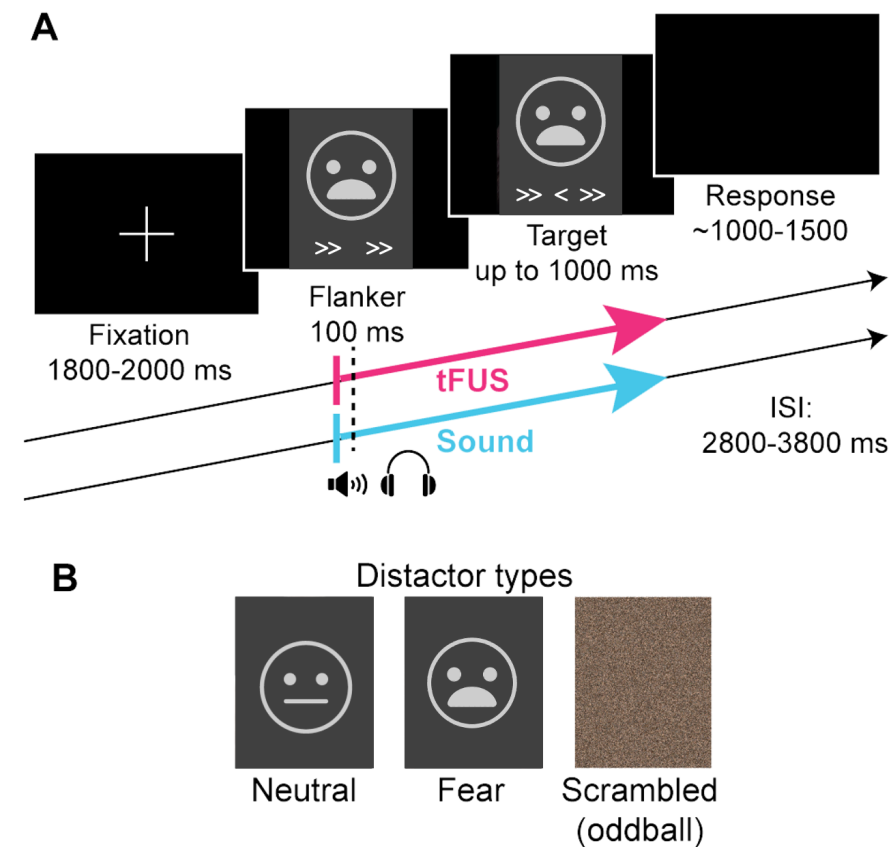

Figure 9. Behavioral task protocol, modified flanker task. (A) Each trial began with a fixation cross after which the flanker arrows appeared overlaid on a face image (vertical dotted line), and were displayed for $100 \mathrm{~ms}$. Sham sound and tFUS stimulation began $28 \mathrm{~ms}$ prior to the face and distractor arrow presentation, and lasted 500ms. The target arrow appeared on the screen until the subject's response, after which a blank screen appeared. The presentation time of the blank screen was adjusted such that the ISI of tFUS stimulation was never to be less than $2800 \mathrm{~ms}$. The example trial displayed is an incongruent flanker trial, but congruent and incongruent trials were presented with equal probability. (B) Three face types were used as distractor images: neutral expressions, fearful expressions, and scrambled face images. Actual face stimuli images used not shown for protection of privacy per medRxiv policy. Scrambled faces were presented as an oddball and represented only 6\% of trials. Related to Figure S6.

\section{Behavioral task protocol: modified flanker paradigm}

Subjects performed a modified version of the Eriksen Flaker task (Eriksen and Eriksen, 1974). The experiment began with 32 practice trials, followed by 100 baseline trials (50 congruent, 50 incongruent) of a simple flanker task with white distractor arrows appearing on a black background for $100 \mathrm{~ms}$, followed by the target arrow. The distractor arrows were either congruent (pointing in the same direction as the middle target arrow: $>>>>>$ ) or incongruent (pointing in opposite direction of the target: $>><>>$ ). Subjects were instructed to respond to the direction of the target arrow via button press with their right hand using the index finger for left and the middle for right. Participants were instructed to respond as quickly as possible without sacrificing accuracy. 
All main experimental trials utilized the protocol outlined in Figure S5. Trials began with a fixation cross presented for 1800 - $2500 \mathrm{~ms}$. Sham sound began in both groups $28 \mathrm{~ms}$ prior to the appearance of the distractor arrows, and the tFUS stimulation (described below) began at the same time. Both the sound and the stimulation had a 500 ms duration. Distractor arrows then appeared overlaid on one of three face types: a face exhibiting a neutral expression, one exhibiting a fearful expression, or a scrambled face (colored static). The fearful and neutral faces appeared with equal frequency, will the scrambled trials occurred only $6 \%$ as an oddball. A total of 420 trials were presented in four blocks. Trials were pseudorandomized with equal number of each trial type, and arrow direction. Trials were controlled such that not more than three consecutive correct responses were pointing in the same direction. Face images were used from both the NimStim (Tottenham et al., 2009) and MaxPlank (young faces only) (Ebner et al., 2010) datasets, were edited to have a black background, masked to include just the face, and resized where necessary to ensure consistency in face size as well as position of nose and eyes. Following the completion of the main trials, subjects completed another 100 trials of post-experiment simple flanker trails (identical to the baseline, simple flanker with black background, no faces, both groups received sham stimulation).

\section{Experimental setup}

Trial order was pre-calculated and experimental presentation content was controlled via python and Psychopy (Peirce, 2007). Analogue photodiodes were used to detect the exact time of screen refresh and stimulus presentation (Figure S6A). This signal was digitized by a Cedrus StimTracker box (Cedrus Corporation) and was EEG system via parallel port connection along with information from the subjects' responses, recorded via a button box. tFUS stimulation was controlled via the experiment computer and the timing recorded via parallel-port connection on the EEG.

\section{Sham}

All subjects wore headphones for presentation of a low volume sound meant to emulate the sound of the tFUS pulses herd through bone conduction. The sound file was creating by combing a very high frequency tone with a square wave at the pulse repetition frequency. The sound was played simultaneously with the onset of the tFUS pulse. In both groups, the tFUS transducer was placed on the head (Figure S6B)

\section{Stimulation planning and neuronavigation}

T1 structural MRI scans were collected on all subjects receiving active tFUS for the purposes of neuronavigation. Images were collected in a Philips Ingenia 3T scanner with a 32-channel head coil, using a 3D MPRAGE sequence $(\mathrm{TR}=2300$ $\mathrm{ms}, \mathrm{TE}=4.5 \mathrm{~ms}, 1 \times 1 \times 1 \mathrm{~mm} 3$ voxels, field of view $240 \times 256 \mathrm{~mm}^{2}, 180$ sagittal slices).

All stimulation targets were planned prior to subject arrival. T1s were processed using Brainsuite by running the cortical extraction sequence and surface volume labeling procedure using the Destrieux atlas (Destrieux et al., 2010). Following this, a 3D mask was created for both the white and gray matter of each subjects' right pars opercularis and anterior insula. These masks were then to assist in planning and visualization of tFUS targets using a Brainsight neuronavigation system (Rogue industries). First, an atlas was created to map the Montreal Neurologic Institute (MNI) coordinate system (Evans et al., 1994) onto each subject's brain by identifying the anterior and posterior commissure, and outer bounds of the cortex. The anatomical mask images were then overlaid on the subject's scan to facilitate anatomical identification in 2D and 3D (Figure 1).

Careful consideration was taken to plot a trajectory target in which the tFUS beam path would overlap with the pars opercularis (BA 44) of the rIFG and the tFUS focus would be primarily be within the frontal operculum and anterior insula (BA 13) (Figure 1, Table S1), while the transducer face would be normal to the surface of the scalp and the beam perpendicular. Significant anatomical variation exists in this region, and therefore individual anatomy, rather than MNI coordinates, was prioritized. 

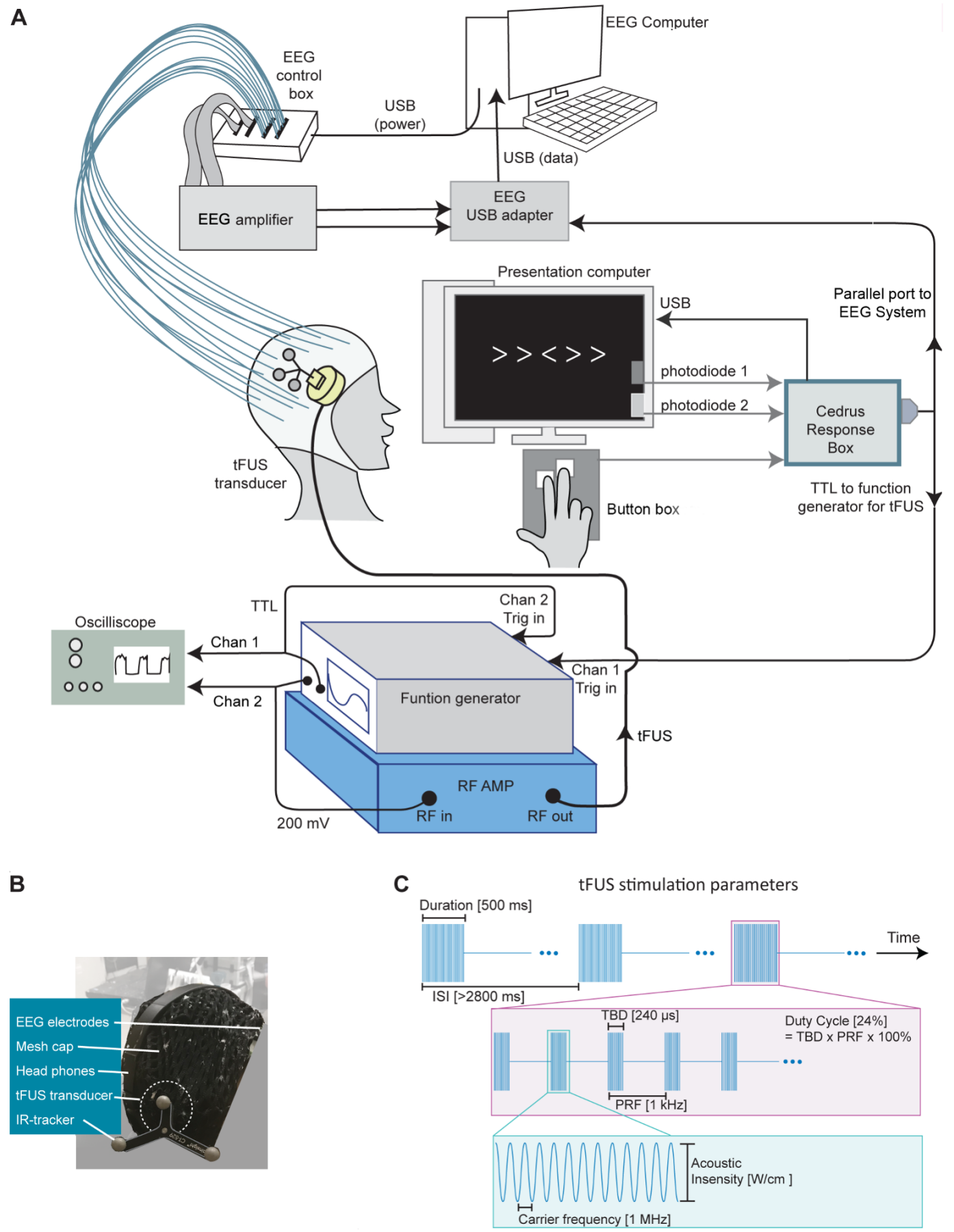

Figure 10. Experimental setup and tFUS parameters. (A) Experimental setup for stimulus presentation and recording. The photodiodes are analog sensors, digitized by the Cedrus response box. Participant responses were recorded via a button box and sent to EEG system via parallel port correction with the photodiode information. Not pictured, headphones from experiment computer, subject tracker, Polaris camera and computer for neuronavigation. (B) Task setup on a single subject. Glasses were used on the subject (not pictured), and the tFUS transducer with attached IR-tracker for neuronavigation. The transducer was held in place with a mesh cap over which the subject wore headphones. (C) tFUS stimulation parameters. Stimulation was repeated at stimulation presentation for each trial in the tFUS group. Acronyms: inter-stimulus interval (ISI), tone burst duration (TBD), pulse repetition frequency (PRF). Abbreviations: Chan- Channel, RF Ampradio frequency amplifier. See also Figure 1 and Figure S5.

An infrared optical tracking system (Polars Vicra, NDI Medical) was used to register the subject's head and tFUS transducer to their MRI using Brainsuite. On the day of the experiment, following EEG setup (described below), glasses with an infrared tracker were placed on the subject in order to register their head in virtual space for neuronavigation. A custom-build housing was 3D-printed for the tFUS transducer to fixate the infrared tracker and to hold acoustically conductive silicone 
(Silicon Solutions) to couple the transducer to the scalp and adjust the penetration depth of the tFUS focus. Ultrasound conductive gel was liberally applied to the scalp and hair to avoid any air bubbles between the traducer face and the skin. Once the transducer was placed in the appropriate area and stimulation location recorded, the transducer was fixed in place with a lightweight, custom-sewn mesh cap to distribute the weight and avoid interference with EEG recording (Figure S6B). The glasses were removed and the transducer remained fixed to the subject's head throughout the experiment. Across all subjects, a mean center coordinates of stimulation were recorded as: $\mathrm{x}=35.4 \pm 13, \mathrm{y}=13.5 \pm 1.2, \mathrm{z}=1.4 \pm 1.7$ (mean \pm SEM, Figure 1, Table S1).

\section{tFUS parameters}

The ultrasound transducer was driven by a $40 \mathrm{~W}$ linear RF amplifier (Legon et al., 2012). The inter-stimulus interval (ISI) between tFUS pulses never exceeded $2800 \mathrm{~ms}$ (Figure S5 and S5). Each stimulus period (duration) was 500ms, and consisted of a tone burst duration (TBD) of $24 \mu \mathrm{s}$, a pulse repetition frequency (PRF) or $1 \mathrm{kHz}$, making a duty cycle of $24 \%$ (Figure S6C). A broadband, single element transducer (Blatek, Inc.) with carrier frequency of $1 \mathrm{MHz}$ was used to optimize transduction across the skull (Tufail et al., 2010). The transducer had a fixed focal depth of $70 \mathrm{~mm}$, focal depth was adjusted by using various width silicone spacers such that actual focus depth ranged from $40-50 \mathrm{~mm}$ from the surface of the scalp to the center point of the beam focus. The focal beam profile has a lateral spatial resolution of $4.9 \mathrm{~mm}^{2}$ and axial spatial resolution of $18 \mathrm{~mm}^{2}$, identical to that described previously (Legon et al., 2014; Mueller et al., 2014). Water tank measurements indicated a max pressure of $1.0 \mathrm{MPa}$ and spatial peak pulse average intensity ( $\mathrm{I}_{\text {sppa) }}$ of $20.4 \mathrm{~W} / \mathrm{cm}^{2}$ at the focus, well below the FDA safety limit for imaging fetal tissue, with the spatial-peak pulse-average intensity $\left(\mathrm{I}_{\mathrm{sppa}}\right) \leq 190 \mathrm{~W} / \mathrm{cm}^{2}$ (Nyborg, 2000), and does not account for power attenuation from the skull.

\section{EEG recording}

Electroencephalography (EEG) data was recorded using a 64-channel ActiCap system (BrainVision) with the standard 10-20 electrode layout. Electrode F8 was removed for placement of the ultrasound transducer. Data was recorded using a sampling rate of $5 \mathrm{kHz}$, resolution of $0.1 \mathrm{~V}$, and band-pass filter of $0.1-100 \mathrm{~Hz}$. The ground was placed at FPz and reference at the left mastoid. Each subject's electrode locations were recorded using a Captrack camera system (BrainVision), with fiducials on the left and right tragus, and nasion.

\section{EEG processing}

Custom scripts were used to process EEG data in MATLAB R2017b (MathWorks) with the utilization of EEGLAB v14.1.1 (Delorme and Makeig, 2004). Raw EEG data was down-sampled to $250 \mathrm{~Hz}$ and high-pass filtered applied at $1 \mathrm{~Hz}$, and notch filtered at $60 \mathrm{~Hz}$. Visual inspection was used to remove glaring artifacts and bad channel. Additionally any channels that exhibited artifacts for more than $25 \%$ of the recording session or with absolute temporal standard deviation greater than five were removed. Interpolation was performed over all removed channels. Data was then re-referenced to the scalp average using individually-recorded electrode locations. Blink, eye-movement, and other glaring artifacts were removed with Independent Components Analysis; on average $3.9 \pm 0.4$ components were removed for each subject. Epoching of the data was then performed around the distractor arrow presentation (target-locked). Trials with late responses or in which reaction time deviated more than three standard deviations from the individual mean, were excluded from analysis.

\section{Event-related potentials}

A pre-stimulus baseline $(200 \mathrm{~ms})$ was subtracted to create event related potential (ERP) data. A linear subtraction of congruent from incongruent ERP data was performed to assess the incongruent-congruent difference potential (Figure 2B). Similarly neutral trial responses were subtracted from fearful ones to create a fear - neutral difference potential (Figure 2).

ERP data was compared across groups at each time point for each trial condition using nonparametric permutation to control for multiple comparisons problems. Statistical $p$ values represent the proportion of 1,000 permutations of randomly shuffled data which produce a $t$ value greater than that calculated by a standard two-tailed, unpaired $t$-test (Maris and Oostenveld, 2007) (statistical significance was considered at $p<0.05$ ). Each trial condition, as well as each face type (fear, neutral) in incongruent - congruent contrast data, and each congruency condition (congruent, incongruent) in fear - neutral contrast data were compared across groups.

In addition, a two-way RM-ANOVA [2 congruency conditions (congruent, incongruent) $\times 2$ face types (neutral, fear)] was used to compare within groups. Additionally for each individual, peak-to-peak amplitude and latency were identified for each the ERP complex, and compared across groups (Table S2 and S3). Additionally nonparametric Friedman's test was used to compare within groups (Table S4).

To analyze response-locked ERPs for correct and error responses, baseline-subtracted data was then time-locked to the response (button press). As with target-locked ERPs, each condition was compared across groups using permutation testing. Additionally, error and correct responses were compared within group using permutation testing (paired rather than unpaired t-tests were used). 


\section{Time-frequency analysis: event-related spectral perturbation}

Time-frequency analysis was performed by computing event-related spectral perturbation (ERSP) data using EEGLAB. Morlet wavelets were used with 3 cycles at the lowest frequency $(1.5 \mathrm{~Hz})$, and increasing linearly to 40 cycles at the highest frequency $(50 \mathrm{~Hz})$. A $500 \mathrm{~ms}$ pre-stimulus baseline was used, and all results are displayed as decibel power above baseline. Statistics were compared at each time and frequency data point. A cluster-based multiple comparisons correction was applied by determining clusters of contiguous significant pixels, $F$ or $t$ values in these clusters were then summed and only clusters greater than 2 standard deviations above the mean were retained. Additionally, false discovery rate (FDR) correction for multiple comparisons was also applied. Specific frequency bands and time windows were selected for display as scalp maps and/or power over time. Selection was based either on peak responses in the ERSP or the results of group level statistical testing.

Response-locked data was analyzed with mixed-measures, two-way RM-ANOVA [2 groups $\times 4$ trial conditions (fear congruent, fear incongruent, neutral congruent, neutral incongruent)] (Figure 2). Similar to ERPs, permutation testing was used to compare all trial conditions across groups, and further quantified within group using RM-ANOVAs [2 congruency conditions (congruent, incongruent) $\times 2$ face types (neutral, fear)] (Figure 3 and 4). Additionally, incongruent - congruent contrast power for each emotion condition (fear, neutral) were compared across groups using permutation testing. Oddball trials were also assessed in a similar way, with RM-ANOVA [2 congruency conditions $\times$ trial frequency conditions (neutral, oddball)] compared within groups (Figure S2). In addition, to disambiguate interaction effects, the results of permutation testing within group across congruent and incongruent conditions are presented for neutral and oddball trials (Figure S2B).

Response-locked correct and error ERSP data were compared both across and within groups using permutation testing (Figure 5). Incongruent - congruent contrast power (linear subtraction of correct from error response) was also compared across groups.

\section{Heart-rate metrics}

A single EEG electrode was removed for placement of the tFUS transducer on the head, and was used to record heartrate (HR) data by placing it below the left clavicle. Raw HR data was first manually processed in MATLAB to ensure correct identification of heart beats peaks. Following this, all HR metrics were calculated using HRVTool (Vollmer, 2015). In add ion to HR, several heart rate variability (HRV) metrics were calculated: R-R interval (time between heart beats), standard deviation of the normal-to-normal heartbeat (SDNN, equation (1)), short term HRV (SD1), long term HRV (SD2), SD1/SD2 ratio, power in the The calculation of SD1 and SD2, which are non-linear measures, can be derived by plotting a Poincaré plot $\left(R R_{n}, R R_{n+1}\right)$ (Karmakar et al., 2009; Vollmer, 2015). SD1 is based on successive differences, and calculated as the standard deviation perpendicular to the identity line, $R R_{n}=-R R_{n+1}$ (equation (2)). SD2 is the standard deviation along the identity line, $R R_{n}=R R-$ ${ }_{n+1}$ (equation (3)), since it is derived from summation of successive RR intervals it is more representative of long-term HRV. HR and HRV metric were compared with a mixed-measures RM-ANOVA [2 groups (Sham, tFUS) $\times 2$ face/stimulation conditions (baseline flanker, flanker with faces and tFUS stimulation)]. One subject in the Sham group was excluded from heart-rate metric analyses due to recording issues.

$$
\begin{aligned}
& S D N N=\sqrt{\frac{1}{n-1} \sum_{i=1}^{n}\left(R R_{i}-\overline{R R}\right)^{2}} \\
& S D 1=\sqrt{1 / 2} \cdot \sigma\left(R R_{i+1}-R R_{i}\right) \\
& S D 2=\sqrt{1 / 2} \cdot \sigma\left(R R_{i+1}+R R_{i}\right)
\end{aligned}
$$

\section{PANAS mood ratings}

To evaluate mood changes, subject were asked to complete the Positive Affect and Negative Affect Schedule (PANAS)(Watson et al., 1988) via REDCap. Mood scales were completed at baseline and immediately following experiment completion. PANAS ratings were summed to deduce positive and negative scores. Ratings for positive valence probes ('interested', 'excited', 'strong', 'enthusiastic', 'proud', 'alert', 'inspired', 'determined', 'attentive', 'active') were summed to create a PANAS positive score while negative valence probes ('depressed', 'upset', 'guilty', 'scared', 'hostile', 'irritable', 'ashamed', 'nervous,' 'afraid') were summed to create PANAS negative score. Scores were compared with a mixed-factor RMANOVA [2 groups $\times 2$ time points (baseline, post-experiment)]. One subject in the insula group was excluded from PANAS metric analyses due to recording issues.

\section{Behavioral responses}

Main trial RT's were baseline-subtracted to removed any individual differences. Each subject' s median baseline RT for congruent and incongruent trials (simple flanker task on black background, both groups received sham stimulation) was used. Baseline congruent RT was subtracted from congruent trials (neutral, fear, and oddball), and likewise for incongruent. 
Groups were then compared using Mann-Whitney U test. Additionally, median RT's were compared within group using Friedman's nonparametric test, post-hocs with Wilcoxon signed-ranks test.

To access conflict adaption, trials were separated by previous trial congruency (congruent, incongruent), and median congruent RT was subtracted from incongruent to calculated the congruency effect in the RT. These were then compared both across and within group.

\section{Post error slowing}

To assess post error slowing, RTs on fear and neutral trials following an error were compared with median RTs in both congruency conditions using Wilcox signed-rank tests. To assess post error slowing across groups, median RT was subtracted from post error RTs and groups were compared using Mann-Whitney U tests.

\section{Data analysis and statistical methods}

Only correct responses were considered for analysis unless indicated as an error response. Trials with late RTs, RTs that deviated more than three standard deviations from the individual mean or were greater than $900 \mathrm{~ms}$, were removed from analysis of behavioral and EEG data.

Statistical analysis was conducted using SPSS Statistics Software SPSS 26.0 (IBM Corporation, Armonk, NY) and EEGLAB v14.1.1. Both parametric (RM-ANOVA), and non-parametric analyze were employed were appropriate and indicated above (between groups: permutation testing, Mann-Whitney U test; within groups: Friedman's test and Wilcox signed-ranks tests). Threshold for statistical significance were set at $p<0.05$. Bonferroni-correction was used for all post-hoc $p$-values. FDR correction for multiple comparisons was applied were indicated. Additionally, a cluster-based multiple comparison correction was employed for time-frequency data in which clusters with cumulative $\mathrm{F}$ or $\mathrm{T}$ value score than 2 standard deviations from the mean were highlighted. When necessary normality was confirmed using Kolmogorov-Smirnov tests and Levene's test for homoscedasticity was used to examine all between group data variance (all $p$ 's $>0.05$ ). 


\section{REFERENCES}

Abboud, H., Berroir, S., Labreuche, J., Orjuela, K., and Amarenco, P. (2006). Insular involvement in brain infarction increases risk for cardiac arrhythmia and death. Annals of Neurology 59, 691-699.

Bensmann, W., Roessner, V., Stock, A.K., and Beste, C. (2018). Catecholaminergic Modulation of Conflict Control Depends on the Source of Conflicts. Int J Neuropsychopharmacol 21, 901-909.

Bernat, E., Nelson, L., and Baskin-Sommers, A. (2012). Time-frequency Theta and Delta measures index separable components of feedback processing. International Journal of Psychophysiology 3, 341.

Billeke, P., Ossandon, T., Perrone-Bertolotti, M., Kahane, P., Bastin, J., Jerbi, K., Lachaux, J.-P., and Fuentealba, P. (2020). Human anterior insula encodes performance feedback and relays prediction error to the medial prefrontal cortex. Cerebral Cortex 30 , 4011-4025.

Boksem, M.A., Meijman, T.F., and Lorist, M.M. (2005). Effects of mental fatigue on attention: an ERP study. Brain Res Cogn Brain Res 25, 107-116.

Bretherton, P.M., Eysenck, M.W., Richards, A., and Holmes, A. (2017). Target and distractor processing and the influence of load on the allocation of attention to task-irrelevant threat. Neuropsychologia.

Cai, W., Ryali, S., Chen, T., Li, C.S.R., and Menon, V. (2014). Dissociable Roles of Right Inferior Frontal Cortex and Anterior Insula in Inhibitory Control: Evidence from Intrinsic and Task-Related Functional Parcellation, Connectivity, and Response Profile Analyses across Multiple Datasets. Journal of Neuroscience 34, 14652-14667.

Carlson, J.M., and Reinke, K.S. (2010). Spatial attention-related modulation of the N170 by backward masked fearful faces. Brain and Cognition 73, 20-27.

Carretié, L. (2014). Exogenous (automatic) attention to emotional stimuli: a review. Cognitive, Affective, \& Behavioral Neuroscience 14, 1228-1258.

Ciccone, A.B., Siedlik, J.A., Wecht, J.M., Deckert, J.A., Nguyen, N.D., and Weir, J.P. (2017). Reminder: RMSSD and SD1 are identical heart rate variability metrics. Muscle \& Nerve 56, 674-678.

Colivicchi, F., Bassi, A., Santini, M., and Caltagirone, C. (2004). Cardiac Autonomic Derangement and Arrhythmias in Right-Sided Stroke With Insular Involvement. Stroke 35, 2094-2098.

Coste, C.P., and Kleinschmidt, A. (2016). Cingulo-opercular network activity maintains alertness. Neuroimage 128, 264-272.

Craig, A.D. (2002). How do you feel? Interoception: the sense of the physiological condition of the body. Nature reviews neuroscience 3,655 .

Craig, A.D. (2004). Human feelings: why are some more aware than others? Trends in Cognitive Sciences 8, 239-241.

Crawford, J.R., and Henry, J.D. (2004). The Positive and Negative Affect Schedule (PANAS): Construct validity, measurement properties and normative data in a large non-clinical sample. British journal of clinical psychology 43, 245-265.

Critchley, H.D., Mathias, C.J., and Dolan, R.J. (2002a). Fear Conditioning in Humans: The Influence of Awareness and Autonomic Arousal on Functional Neuroanatomy. Neuron 33, 653-663.

Critchley, H.D., Melmed, R.N., Featherstone, E., Mathias, C.J., and Dolan, R.J. (2002b). Volitional control of autonomic arousal: a functional magnetic resonance study. Neuroimage 16, 909-919.

Critchley, H.D., Wiens, S., Rotshtein, P., and Dolan, R.J. (2004). Neural systems supporting interoceptive awareness. Nature neuroscience $7,189$.

Cuthbert, B.N., Schupp, H.T., Bradley, M.M., Birbaumer, N., and Lang, P.J. (2000). Brain potentials in affective picture processing: covariation with autonomic arousal and affective report. Biological Psychology 52, 95-111. 
de Morree, H.M., Rutten, G.-J., Szabó, B.M., Sitskoorn, M.M., and Kop, W.J. (2016). Effects of Insula Resection on Autonomic Nervous System Activity. Journal of Neurosurgical Anesthesiology 28, 153-158.

Debener, S., Ullsperger, M., Siegel, M., Fiehler, K., Von Cramon, D.Y., and Engel, A.K. (2005). Trial-by-trial coupling of concurrent electroencephalogram and functional magnetic resonance imaging identifies the dynamics of performance monitoring. Journal of Neuroscience 25, 11730-11737.

Delorme, A., and Makeig, S. (2004). EEGLAB: an open source toolbox for analysis of single-trial EEG dynamics including independent component analysis. Journal of neuroscience methods 134, 9-21.

Dennis, T.A., and Chen, C.-C. (2007). Neurophysiological mechanisms in the emotional modulation of attention: The interplay between threat sensitivity and attentional control. Biological Psychology 76, 1-10.

Destrieux, C., Fischl, B., Dale, A., and Halgren, E. (2010). Automatic parcellation of human cortical gyri and sulci using standard anatomical nomenclature. Neuroimage 53,1-15.

Dosenbach, N.U., Fair, D.A., Cohen, A.L., Schlaggar, B.L., and Petersen, S.E. (2008). A dual-networks architecture of top-down control. Trends in cognitive sciences 12, 99-105.

Dosenbach, N.U., Visscher, K.M., Palmer, E.D., Miezin, F.M., Wenger, K.K., Kang, H.C., Burgund, E.D., Grimes, A.L., Schlaggar, B.L., and Petersen, S.E. (2006). A core system for the implementation of task sets. Neuron 50, 799-812.

Ebner, N.C., Riediger, M., and Lindenberger, U. (2010). FACES--a database of facial expressions in young, middle-aged, and older women and men: development and validation. Behav Res Methods 42, 351-362.

Eckert, M.A., Menon, V., Walczak, A., Ahlstrom, J., Denslow, S., Horwitz, A., and Dubno, J.R. (2009). At the heart of the ventral attention system: the right anterior insula. Human brain mapping 30, 2530-2541.

Eimer, M. (2000). Attentional modulations of event-related brain potentials sensitive to faces. Cognitive Neuropsychology 17, 103-116.

Eriksen, B.A., and Eriksen, C.W. (1974). Effects of noise letters upon the identification of a target letter in a nonsearch task. Perception \& psychophysics 16, 143-149.

Evans, A.C., Kamber, M., Collins, D., and MacDonald, D. (1994). An MRI-based probabilistic atlas of neuroanatomy. In Magnetic resonance scanning and epilepsy (Springer), pp. 263-274.

Fallahi, M., Motamedzade, M., Heidarimoghadam, R., Soltanian, A.R., and Miyake, S. (2016). Effects of mental workload on physiological and subjective responses during traffic density monitoring: A field study. Applied Ergonomics 52, 95-103.

Fini, M., and Tyler, W.J. (2017). Transcranial focused ultrasound: a new tool for non-invasive neuromodulation. International Review of Psychiatry, 1-10.

Fischer, A.G., Nigbur, R., Klein, T.A., Danielmeier, C., and Ullsperger, M. (2018). Cortical beta power reflects decision dynamics and uncovers multiple facets of post-error adaptation. Nature Communications 9.

Gaebler, M., Daniels, J.K., Lamke, J.-P., Fydrich, T., and Walter, H. (2013). Heart rate variability and its neural correlates during emotional face processing in social anxiety disorder. Biological Psychology 94, 319-330.

Grace, A.A. (1995). The tonic/phasic model of dopamine system regulation: its relevance for understanding how stimulant abuse can alter basal ganglia function. Drug and Alcohol Dependence 37, 111-129.

Guzik, P., Piskorski, J., Krauze, T., Bychowiec, B., Wesseling, K., Schneider, R., Girgus, P., Wykrtowicz, A., and Wysocki, H. (2005). Numerical descriptors of Poincaré plots analysis of RR intervals are related to baroreflex sensitivity and hemodynamic parameters in healthy people. Folia Cardiol 12, 56-59.

Hajcak, G., McDonald, N., and Simons, R.F. (2003). Anxiety and error-related brain activity. Biological Psychology 64, 77-90.

Harper, J., Malone, S.M., and Bernat, E.M. (2014). Theta and delta band activity explain N2 and P3 ERP component activity in a go/no-go task. Clinical Neurophysiology 125, 124-132.

Modulation of Emotionally Distracted Networks by tFUS: aIns/fO targeted effects. 
Iannaccone, R., Hauser, T.U., Staempfli, P., Walitza, S., Brandeis, D., and Brem, S. (2015). Conflict monitoring and error processing: New insights from simultaneous EEG-fMRI. NeuroImage 105, 395-407.

Jabbi, M., and Keysers, C. (2008). Inferior frontal gyrus activity triggers anterior insula response to emotional facial expressions. Emotion 8, 775-780.

Kanske, P., Heissler, J., Schönfelder, S., Bongers, A., and Wessa, M. (2011). How to Regulate Emotion? Neural Networks for Reappraisal and Distraction. Cerebral Cortex 21, 1379-1388.

Kapp, B.S., Schwaber, J.S., and Driscoll, P.A. (1985). The organization of insular cortex projections to the amygdaloid central nucleus and autonomic regulatory nuclei of the dorsal medulla. Brain Research 360, 355-360.

Karmakar, C.K., Khandoker, A.H., Gubbi, J., and Palaniswami, M. (2009). Complex correlation measure: a novel descriptor for Poincaré plot. Biomedical engineering online $8,17$.

Klein, T.A., Endrass, T., Kathmann, N., Neumann, J., von Cramon, D.Y., and Ullsperger, M. (2007). Neural correlates of error awareness. NeuroImage 34, 1774-1781.

Klumpp, H., Angstadt, M., and Phan, K.L. (2012). Insula reactivity and connectivity to anterior cingulate cortex when processing threat in generalized social anxiety disorder. Biological Psychology 89, 273-276.

Knyazev, G.G. (2007). Motivation, emotion, and their inhibitory control mirrored in brain oscillations. Neuroscience \& Biobehavioral Reviews 31, 377-395.

Legon, W., Rowlands, A., Opitz, A., Sato, T.F., and Tyler, W.J. (2012). Pulsed Ultrasound Differentially Stimulates Somatosensory Circuits in Humans as Indicated by EEG and fMRI. PLoS ONE 7, 1-14.

Legon, W., Sato, T.F., Opitz, A., Mueller, J., Barbour, A., Williams, A., and Tyler, W.J. (2014). Transcranial focused ultrasound modulates the activity of primary somatosensory cortex in humans. Nat Neurosci 17, 322-329.

Levy, B.J., and Wagner, A.D. (2011). Cognitive control and right ventrolateral prefrontal cortex: reflexive reorienting, motor inhibition, and action updating. Ann N Y Acad Sci 1224, 40-62.

Maris, E., and Oostenveld, R. (2007). Nonparametric statistical testing of EEG- and MEG-data. J Neurosci Methods 164, 177-190.

Meng, X., Gao, S., Liu, W., Zhang, L., Suo, T., and Li, H. (2019). The Childhood Maltreatment Modulates the Impact of Negative Emotional Stimuli on Conflict Resolution. Frontiers in Psychology 10.

Moore, A., Gruber, T., Derose, J., and Malinowski, P. (2012). Regular, brief mindfulness meditation practice improves electrophysiological markers of attentional control. Frontiers in human neuroscience 6.

Mueller, J., Legon, W., Opitz, A., Sato, T.F., and Tyler, W.J. (2014). Transcranial focused ultrasound modulates intrinsic and evoked EEG dynamics. Brain stimulation 7, 900-908.

Neumann, M.F., Mohamed, T.N., and Schweinberger, S.R. (2011). Face and object encoding under perceptual load: ERP evidence. NeuroImage 54, 3021-3027.

Nyborg, W.L. (2000). Biological effects of ultrasound: development of safety guidelines. Part I: personal histories. Ultrasound in medicine \& biology 26, 911-964.

Papazacharias, A., Taurisano, P., Fazio, L., Gelao, B., Di Giorgio, A., Lo Bianco, L., Quarto, T., Mancini, M., Porcelli, A., Romano, R., et al. (2015). Aversive emotional interference impacts behavior and prefronto-striatal activity during increasing attentional control. Front Behav Neurosci 9, 97.

Park, G., Van Bavel, J.J., Vasey, M.W., and Thayer, J.F. (2013). Cardiac vagal tone predicts attentional engagement to and disengagement from fearful faces. Emotion 13, 645-656.

Park, G., Vasey, M.W., Van Bavel, J.J., and Thayer, J.F. (2014). When tonic cardiac vagal tone predicts changes in phasic vagal tone: the role of fear and perceptual load. Psychophysiology 51, 419-426. 
Peirce, J.W. (2007). PsychoPy—psychophysics software in Python. Journal of neuroscience methods 162, 8-13.

Phelps, E.A., O'Connor, K.J., Gatenby, J.C., Gore, J.C., Grillon, C., and Davis, M. (2001). Activation of the left amygdala to a cognitive representation of fear. Nature neuroscience 4, 437-441.

Righart, R., and de Gelder, B. (2006). Context Influences Early Perceptual Analysis of Faces-An Electrophysiological Study. Cerebral Cortex 16, 1249-1257.

Saleh, T.M., and Connell, B.J. (1998). Role of the insular cortex in the modulation of baroreflex sensitivity. American Journal of Physiology-Regulatory, Integrative and Comparative Physiology 274, R1417-R1424.

Sanguinetti, J.L., Hameroff, S., Smith, E.E., Sato, T., Daft, C.M.W., Tyler, W.J., and Allen, J.J.B. (2020). Transcranial Focused Ultrasound to the Right Prefrontal Cortex Improves Mood and Alters Functional Connectivity in Humans. Frontiers in Human Neuroscience 14.

Shafer, A.T., Matveychuk, D., Penney, T., O'Hare, A.J., Stokes, J., and Dolcos, F. (2012). Processing of emotional distraction is both automatic and modulated by attention: evidence from an event-related fMRI investigation. Journal of cognitive neuroscience $24,1233-1252$.

Shaffer, F., McCraty, R., and Zerr, C.L. (2014). A healthy heart is not a metronome: an integrative review of the heart's anatomy and heart rate variability. Frontiers in Psychology 5.

Shi, C., and Davis, M. (1999). Pain pathways involved in fear conditioning measured with fear-potentiated startle: lesion studies. Journal of Neuroscience 19, 420-430.

Smith, N.K., Cacioppo, J.T., Larsen, J.T., and Chartrand, T.L. (2003). May I have your attention, please: Electrocortical responses to positive and negative stimuli. Neuropsychologia 41,171-183.

Spangler, D.P., and McGinley, J.J. (2020). Vagal Flexibility Mediates the Association Between Resting Vagal Activity and Cognitive Performance Stability Across Varying Socioemotional Demands. Frontiers in Psychology 11.

Sridharan, D., Levitin, D.J., and Menon, V. (2008). A critical role for the right fronto-insular cortex in switching between centralexecutive and default-mode networks. Proc Natl Acad Sci U S A 105, 12569-12574.

Tang, Y.-Y., Lu, Q., Feng, H., Tang, R., and Posner, M.I. (2015). Short-term meditation increases blood flow in anterior cingulate cortex and insula. Frontiers in Psychology 6.

Taylor, K.S., Seminowicz, D.A., and Davis, K.D. (2009). Two systems of resting state connectivity between the insula and cingulate cortex. Human brain mapping 30, 2731-2745.

Teper, R., and Inzlicht, M. (2013). Meditation, mindfulness and executive control: the importance of emotional acceptance and brain-based performance monitoring. Social cognitive and affective neuroscience 8, 85-92.

Thayer, J.F., Ahs, F., Fredrikson, M., Sollers, J.J., 3rd, and Wager, T.D. (2012). A meta-analysis of heart rate variability and neuroimaging studies: implications for heart rate variability as a marker of stress and health. Neurosci Biobehav Rev 36, 747756.

Tottenham, N., Tanaka, J.W., Leon, A.C., McCarry, T., Nurse, M., Hare, T.A., Marcus, D.J., Westerlund, A., Casey, B.J., and Nelson, C. (2009). The NimStim set of facial expressions: judgments from untrained research participants. Psychiatry Res 168, 242-249.

Tufail, Y., Matyushov, A., Baldwin, N., Tauchmann, M.L., Georges, J., Yoshihiro, A., Tillery, S.I.H., and Tyler, W.J. (2010). Transcranial pulsed ultrasound stimulates intact brain circuits. Neuron 66, 681-694.

van Driel, J., Ridderinkhof, K.R., and Cohen, M.X. (2012). Not All Errors Are Alike: Theta and Alpha EEG Dynamics Relate to Differences in Error-Processing Dynamics. Journal of Neuroscience 32, 16795-16806.

Vollmer, M. (2015). A robust, simple and reliable measure of heart rate variability using relative RR intervals. Paper presented at: 2015 Computing in Cardiology Conference (CinC) (IEEE). 
Watson, D., Clark, L.A., and Tellegen, A. (1988). Development and validation of brief measures of positive and negative affect: the PANAS scales. Journal of personality and social psychology 54,1063.

Wessel, J.R., and Aron, A.R. (2017). On the globality of motor suppression: unexpected events and their influence on behavior and cognition. Neuron 93, 259-280.

Wessel, J.R., Danielmeier, C., Morton, J.B., and Ullsperger, M. (2012). Surprise and error: common neuronal architecture for the processing of errors and novelty. Journal of Neuroscience 32, 7528-7537.

Williams, L.M., Brown, K.J., Das, P., Boucsein, W., Sokolov, E.N., Brammer, M.J., Olivieri, G., Peduto, A., and Gordon, E. (2004). The dynamics of cortico-amygdala and autonomic activity over the experimental time course of fear perception. Cognitive Brain Research 21, 114-123.

Zaki, J., Davis, J.I., and Ochsner, K.N. (2012). Overlapping activity in anterior insula during interoception and emotional experience. NeuroImage 62, 493-499.

\section{Acknowledgements}

We would like to thank Marco Santello of the School of Biological and Health Systems Engineering for the use of his lab and equipment, as well as many thoughtful discussions during these studies.

\section{Disclosures}

WJT is an equity holding member of IST, LLC a commercially active neurotechnology company. WJT is also the inventor on USA and international issued and pending patents related to the methods and devices used in this study for the modulation of brain activity by focused ultrasound. 


\section{Supplemental Materials}

\section{Supplemental Figures}

A

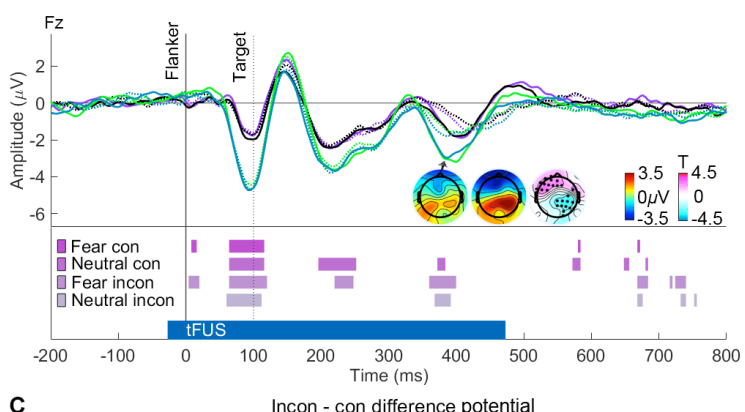

C
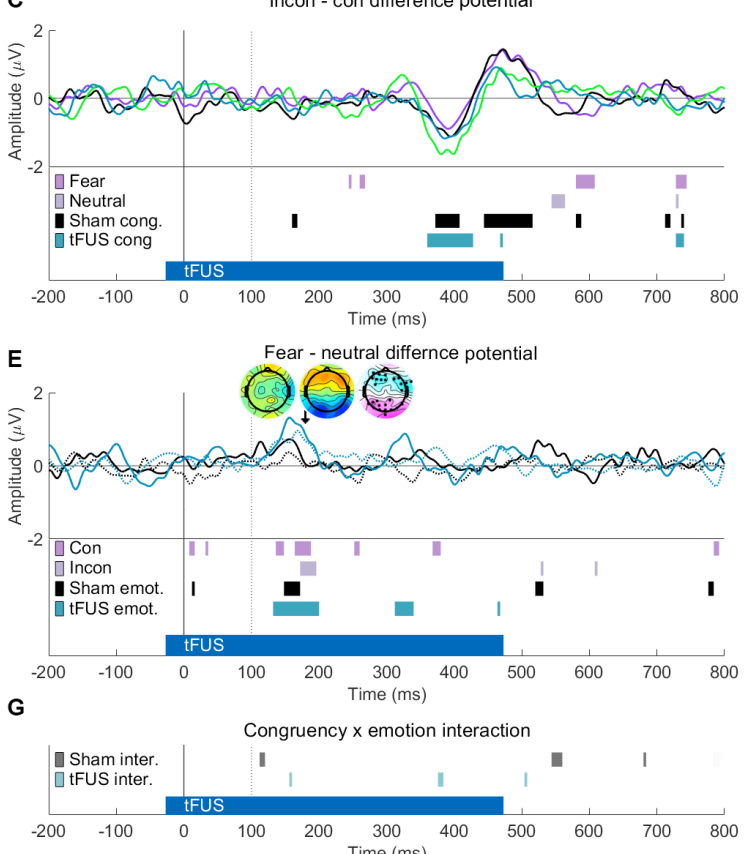

B
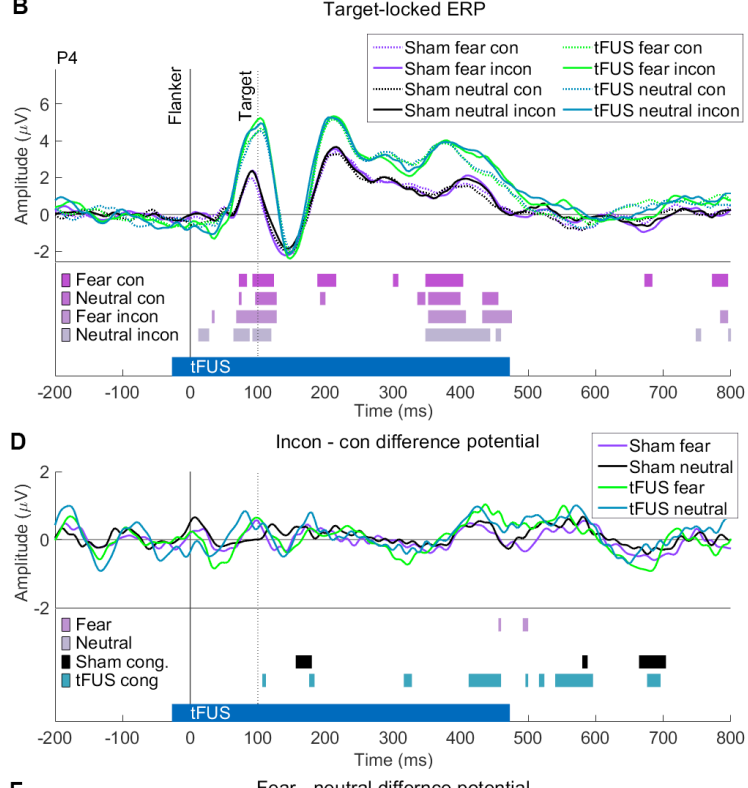

$\mathbf{F}$
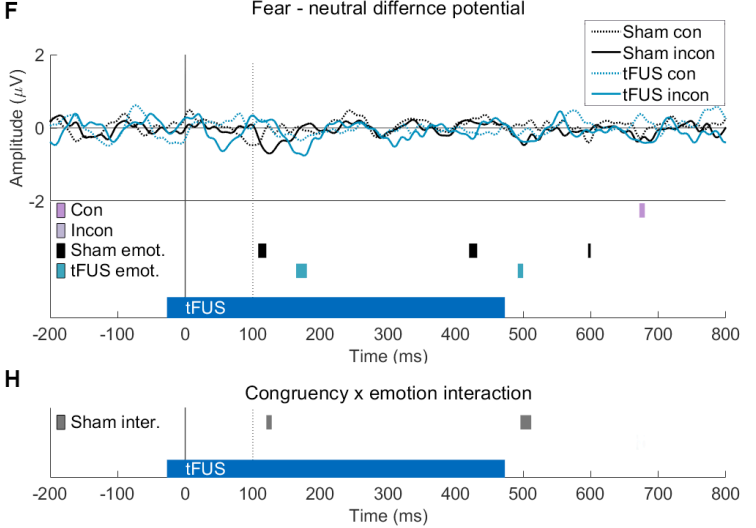

Figure S1 Target-locked ERP for frontal and parietal electrodes. (A-B) Displays ERPs at Fz (A) and P4 (B) for each group, emotion, and congruency condition. Significant differences across groups for condition are displayed in the lower panel (permutation testing, $p<0.05$ ). tFUS and Sham sound stimulation period marked with blue bar at the bottom of the panel (tFUS). In A, scalp maps show potential for Sham (left), tFUS (middle), and the results of permutation testing across groups (right) for the fear incongruenct condition, significant electrodes marked with a black dot. (C-D) Subtraction of congruent from incongruent ERPs to make a difference potential for Fz (C) and P4 (D). Lower panel displays significant differences across groups for fear and neutral conditions. Below this, main effect of congruency (RM-ANOVA) is displayed for each group (Sham, tFUS). (E-F) Subtraction of neutral from fear ERPs to produce difference potential at Fz (E) and P4 (F). Lower panel displays significant differences across groups for each congruency condition (con, incon), as well as significant main effect of emotion for each group. (G-H) Congruency $\times$ emotion interaction effect for each group at Fz (G) and P4 (H). Abbreviations: con: congruent, incon: incongruent. Related to Figure 2. 
A
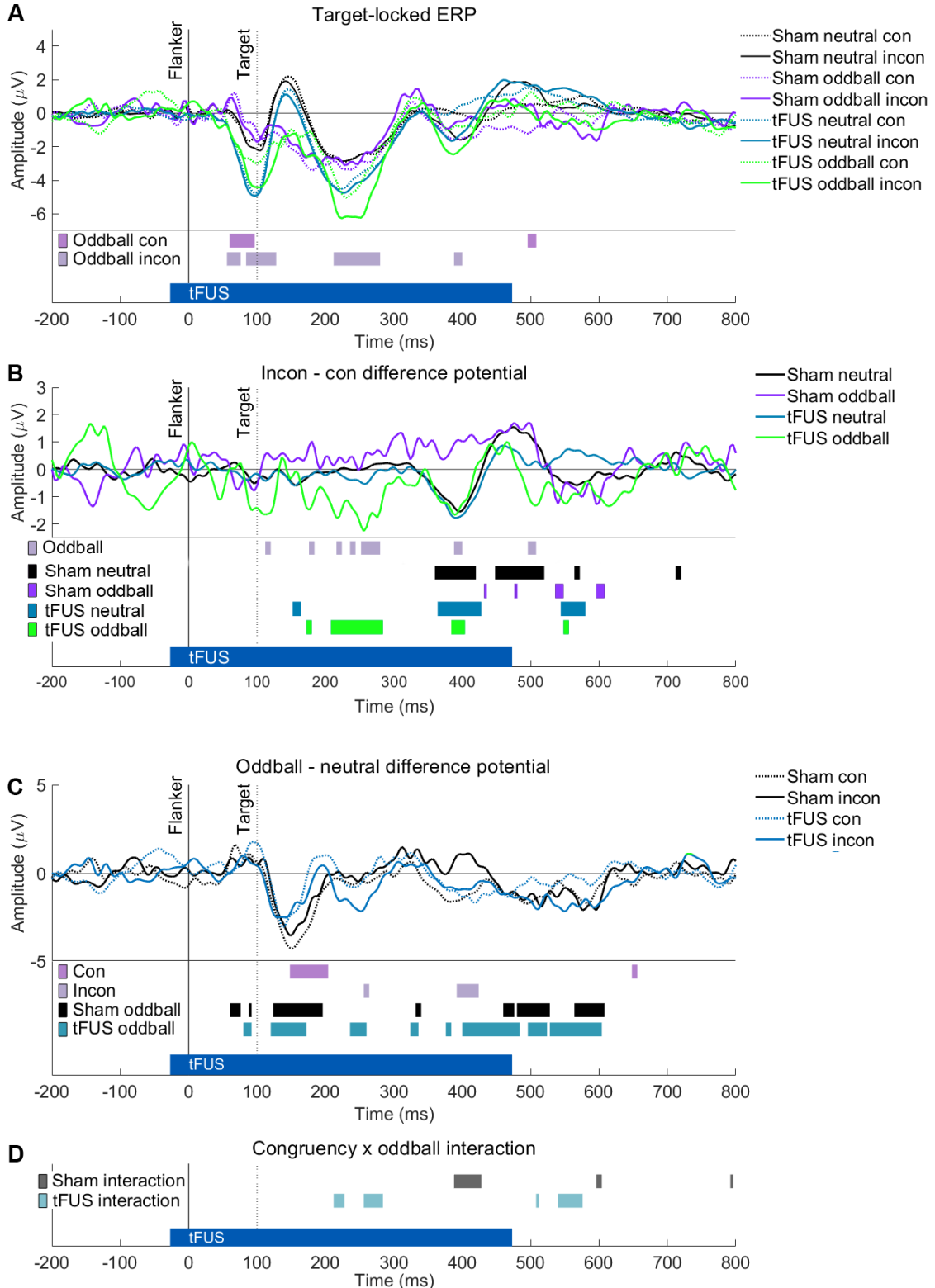

Figure S2 Target-locked ERP for oddball trial response at FCz. (A) Displays ERPs at FCz for each group for neutral and oddball trials (scrambled images, $6 \%$ of trials). Both congruent and incongruent trial responses are displayed. Neutral trial data is duplicated from Figure 2 for comparison purposes. Significant differences across groups for each oddball condition (oddball con, oddball incon) are displayed in the lower panel (permutation testing, $p<0.05$ ). tFUS and Sham sound stimulation period marked with blue bar at the bottom of the panel (tFUS). (B) Subtraction of congruent from incongruent potential to make a difference potential. Lower panel displays significant differences across groups for the oddball condition. Below this, permutation testing across congruent and incongruent trials for each condition are displayed for each group (Sham neutral, Sham oddball, tFUS neutral, tFUS oddball). (C) Subtraction of neutral from fear potential to make difference potential. Lower panel displays significant differences across groups for each congruency condition (con, incon), as well as significant main effect of oddball for each group (RM-ANOVA). (D) Congruency $\times$ oddball interaction effect for each group (RM-ANOVA). Abbreviations: con: congruent, incon: incongruent. See also Figure 2. 

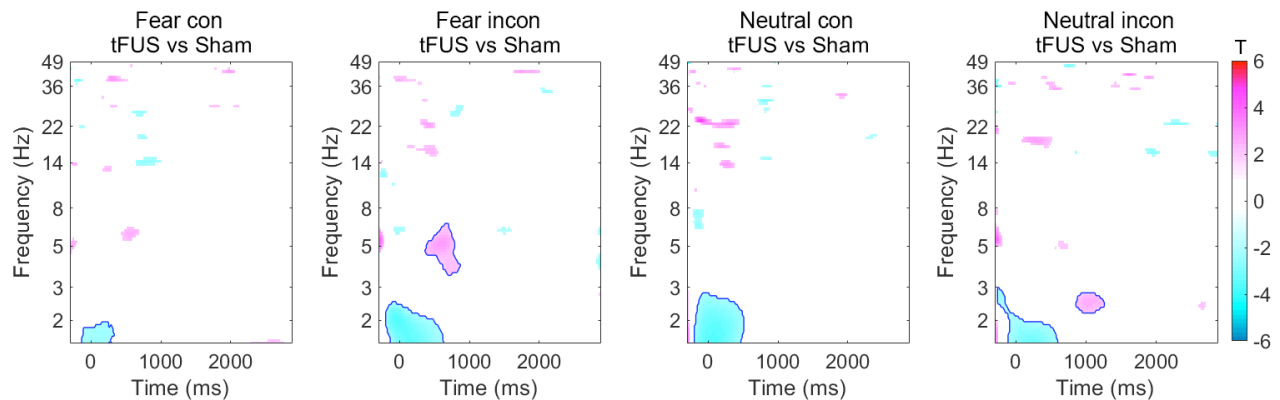

Figure S3. Group comparison of ERSP data at FCz. Displays t-values from permutation testing across groups for each trial condition, only $p<0.05$ displayed. Positive $t$ values indicate power in the Sham group $>\mathrm{tFUS}$, negative indicate Sham $<\mathrm{tFUS}$. Blue outline indicates areas that survive cluster-based thresholding. Related to Figure 3 and Figure S4. See also Figure S5.
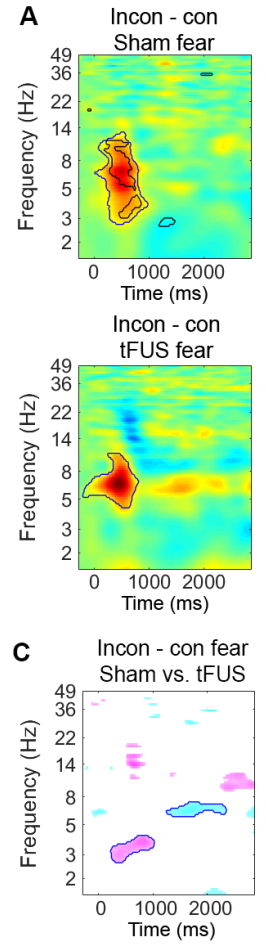
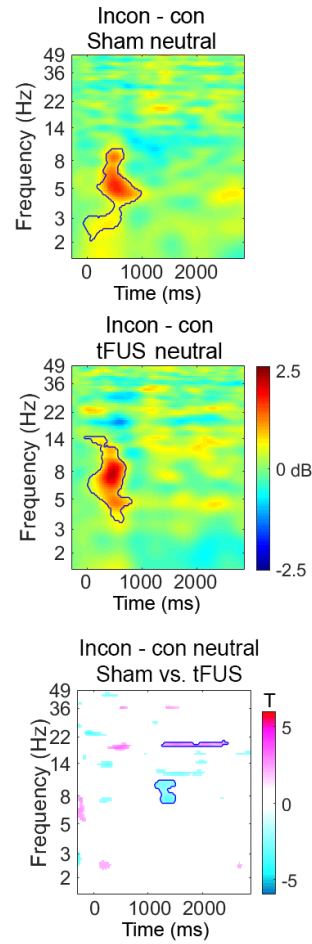
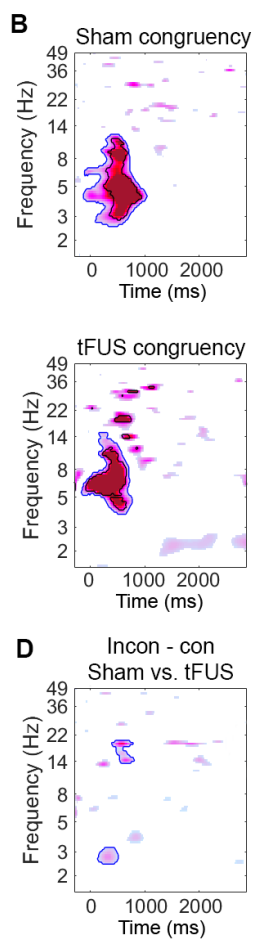
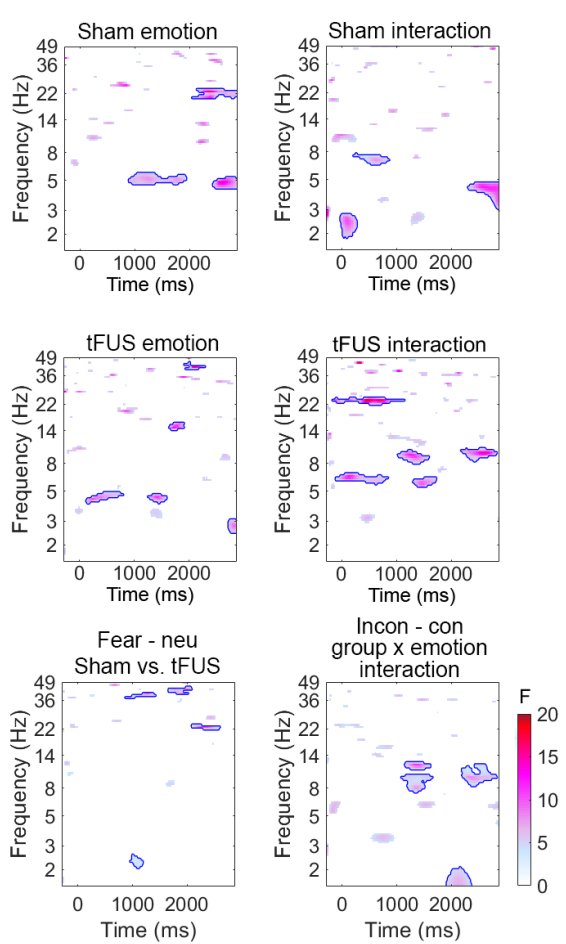
are represented by blue line encircles ( $p<0.05$, cluster-based thresholding), and black line (FDR correction). (B) F-values from RM-ANOVA performed within groups. Left column the main effect of congruency (incon, con), middle the main effect of face emotion (fear, neutral), and right the congruency $\times$ emotion interaction effect. Only $p<0.05$ displayed; blue line: cluster-based thresholding, black line: FDR correction. (C) Permutation testing performed across groups for incongruent - congruent contrast data displayed in A, and separated by face emotion (fear, neutral). (D) Group comparisons: left plot displays F-values from the main effect of group for the incon-con contrast power displayed in A (RM-ANOVA). Middle displays the main effect of group for fear - neutral contrast power. Right-most plot displays the group $\times$ emotion interaction effect for incon - con contrast power. Related to Figure 3 and Figure S3. See also Figure S5. 

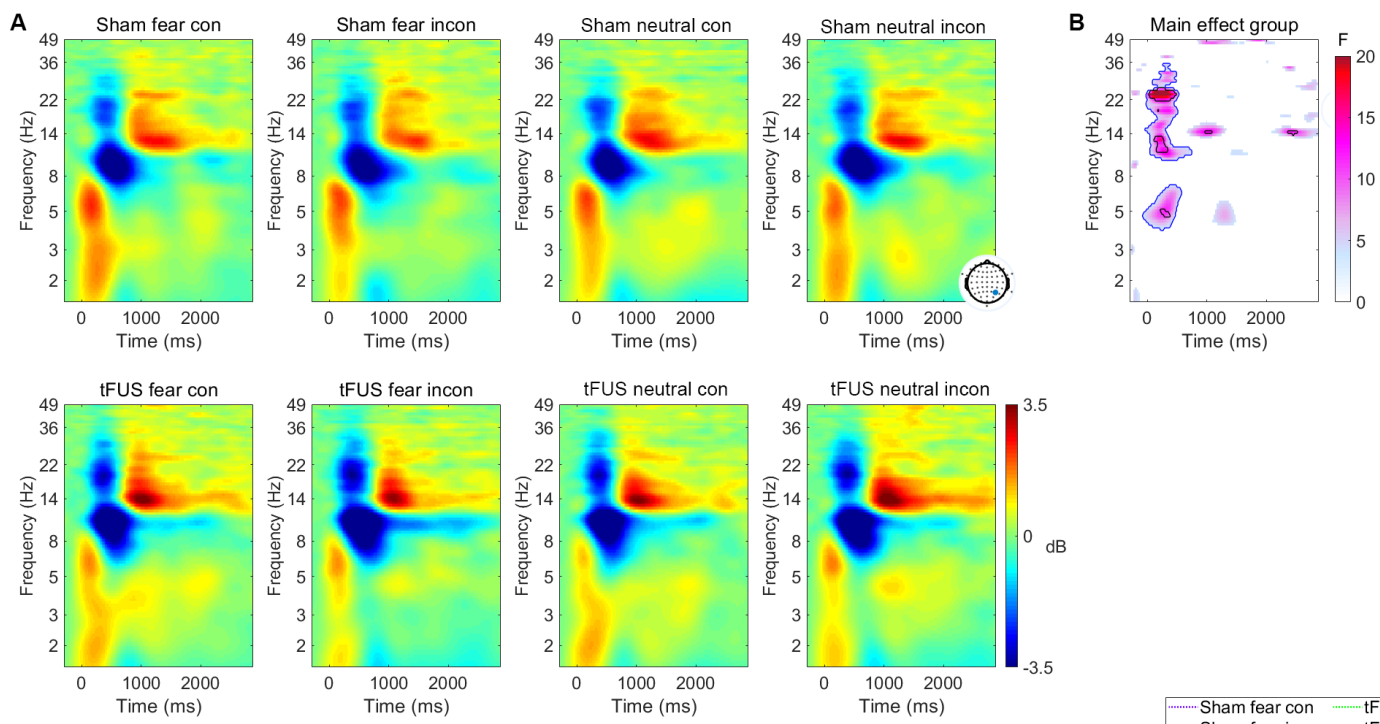

C
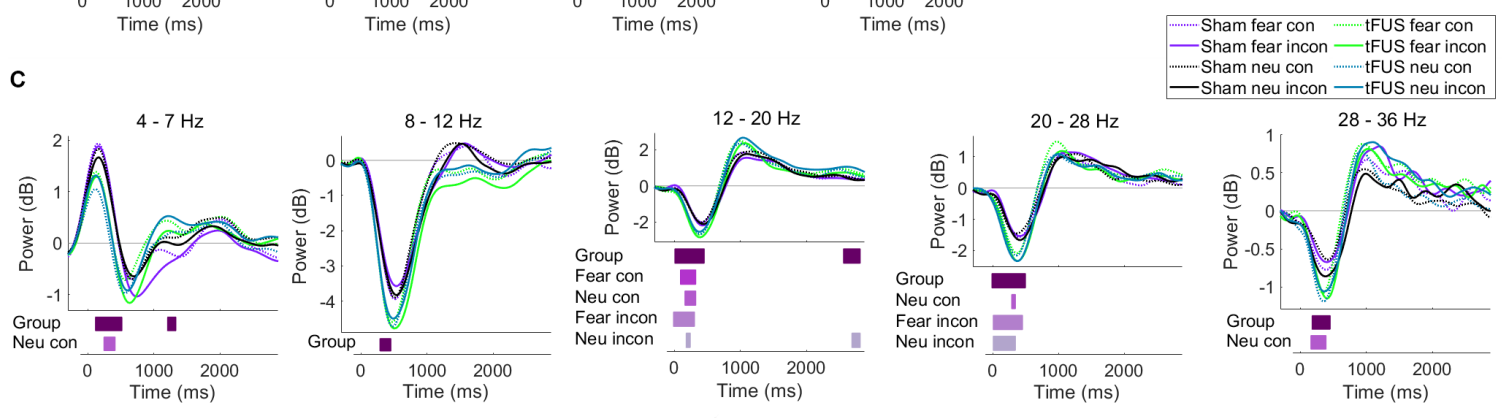

D
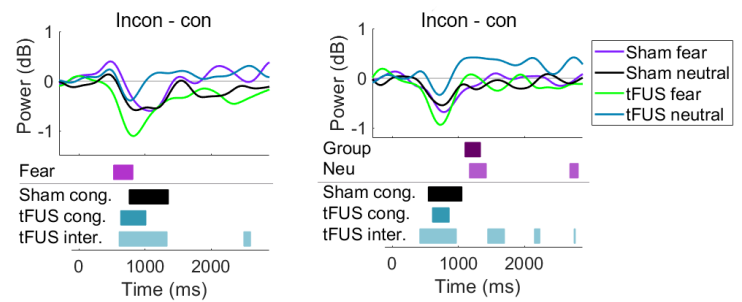

Figure S5. ERSP data at P4. (A) ERSP data at P4 for each group and condition (dB power over baseline). (B) Main effect of group (RMANOVA) of ERSP data displayed in A (F-values). Only $p<0.05$ displayed; blue line: cluster-based thresholding, black line: FDR correction. (C) Power over time in various frequency bands. Lower panel displays significant main effect of group (RM-ANOVA), as well as significant differences in each condition (permutation testing). (D) Incongruent - congruent contrast power for 8-12 Hz (left) and 12-20 Hz (right) frequency bands. Lower panel displays significant main effect of group (RM-ANOVA), and significant differences across groups in each condition (permutation testing). Additionally, significant main effect of congruency (cong.) and congruency $\times$ emotion interaction (inter.) are displayed below for each group (within groups: RM-ANOVA). 

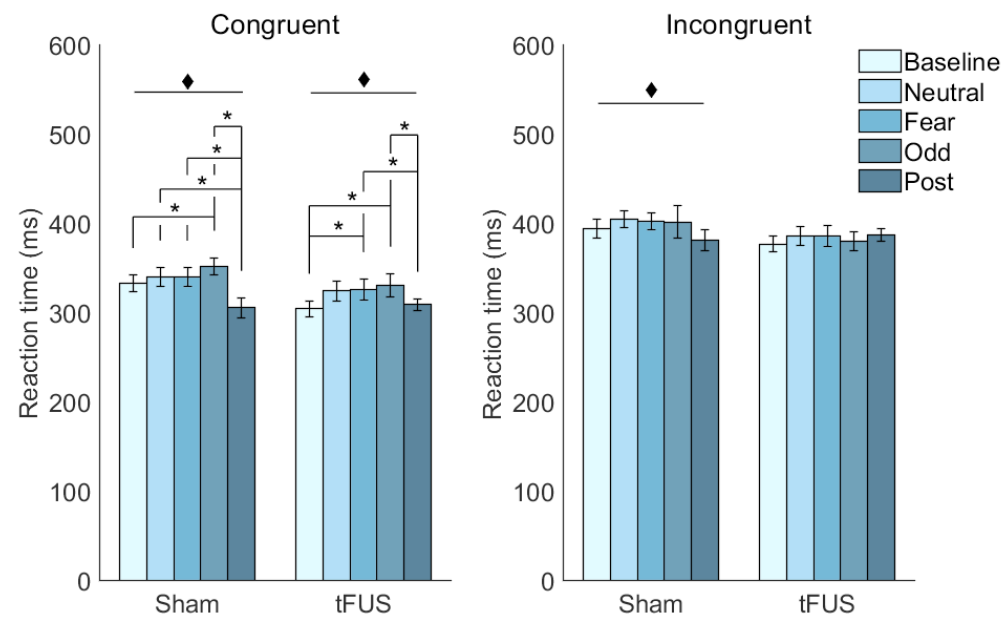

Figure S6. Within-group RT analysis. Median RTs for each trial condition (baseline: simple flanker, no faces, no stimulation), neutral, fear and oddball faces with stimulation, and post-experiment trials: identical to baseline). RTs displayed by group for congruent trials on the left, and incongruent trials on the right. ( indicates significant difference across conditions, Friedman's non-parametric test; $*$ p < 0.05 , Bonferroni-correct post-hoc tests). See also Figure 1, Figure S3.

A

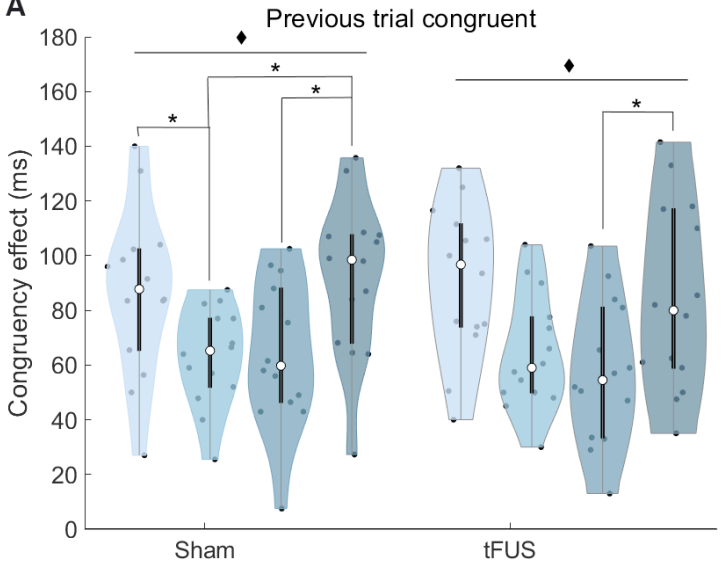

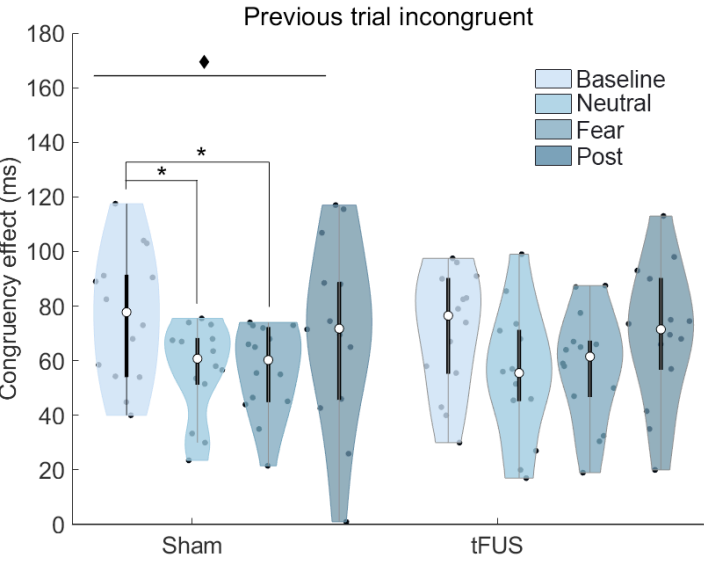

Figure S7. Conflict adaption: congruency effect in reaction time separated by previous trial congruency face trial conditions compared within group. (A) Violin plots display congruency effect by group (median incongruent RT minus congruent RT) for trials in which the previous trial was congruent (left plot) or incongruent (right plot). Trial face types (baseline, neutral, fear, post-stimulation) are compared within group. White dots indicate median, and thick dark-grey bars indicate first and thirds quartiles of the data. ( indicates significant difference across conditions, Friedman's non-parametric test; * p < 0.05, Bonferroni-corrected post-hoc tests). See also Figure S2. 

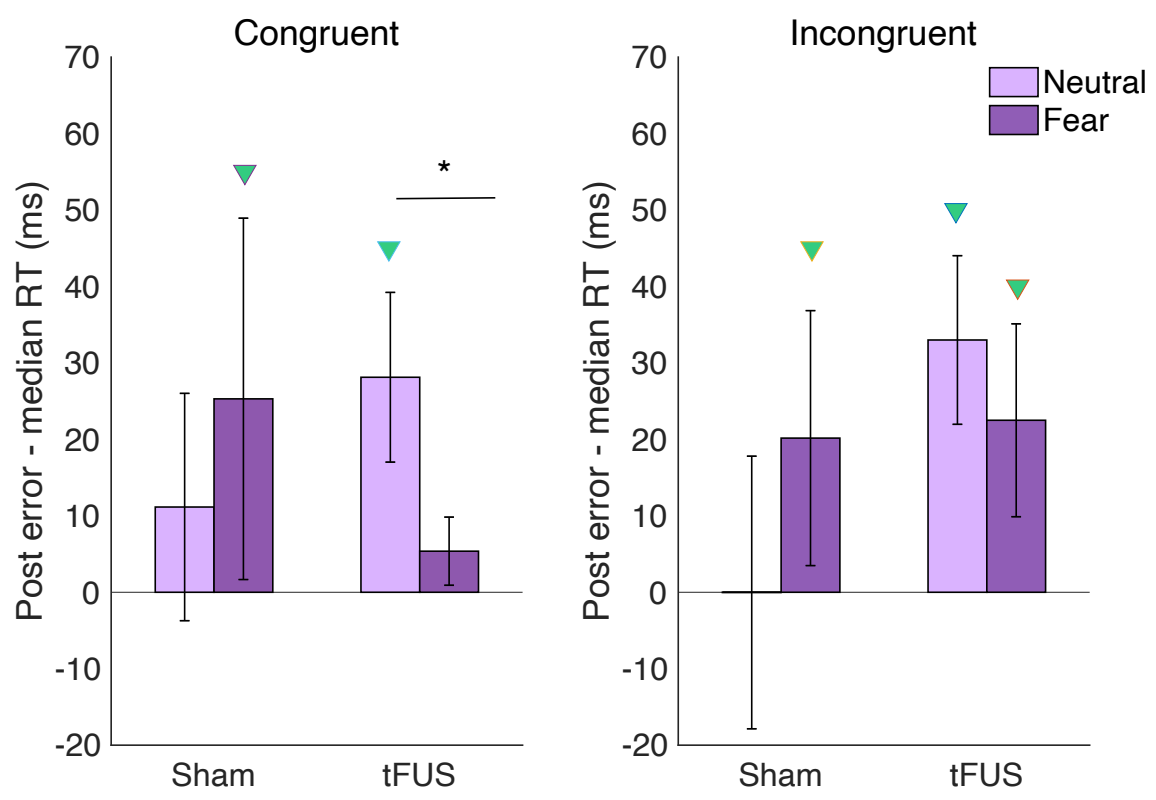

Figure S8. Post error slowing. RTs post error trial minus each subjects' median RT for that condition. Green triangle indicates the posterror RTs are significantly slower than median RTs. Significance within group across emotions indicated with $\left({ }^{*} p<0.05\right)$. Note in both congruent and incongruent post-error trials, the sham group exhibited significant slowing for fear but not neutral trials. In the tFUS group all post error conditions are significantly slower than regular trials, except the fear congruent condition. And there was a significant difference between post error delay in neural and fear congruent trials in the tFUS groups.
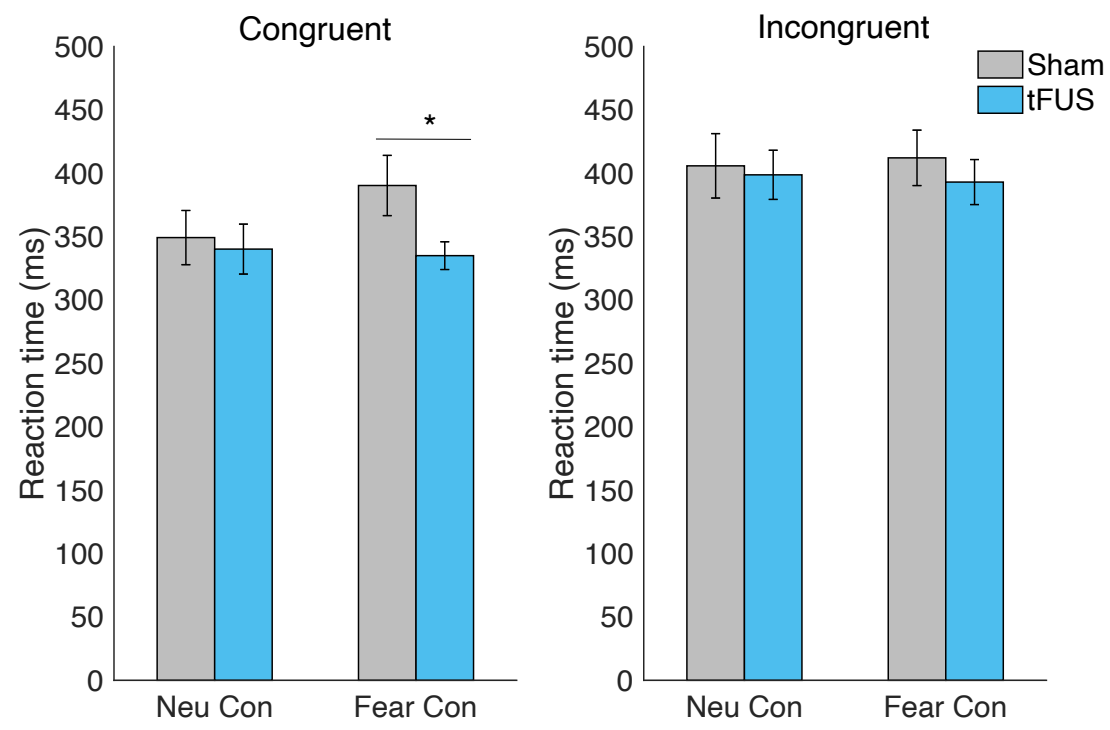

Figure S9. Post Error RTs. Significant differences across groups marked with $\left({ }^{*} p<0.05\right)$.

\section{Supplemental Tables}

\section{Table S1. Stimulation locations for each subject.}




\begin{tabular}{llll}
\hline Subject & $\mathbf{x}$ & $\mathbf{y}$ & $\mathbf{z}$ \\
\hline 1 & 26 & 16 & -7 \\
2 & 38 & 11 & -3 \\
3 & 41 & 9 & 2 \\
4 & 40 & 12 & 0 \\
5 & 33 & 7 & 14 \\
6 & 36 & 13 & -4 \\
7 & 44 & 17 & -3 \\
8 & 35 & 10 & 5 \\
9 & 32 & 17 & 3 \\
10 & 39 & 25 & -5 \\
11 & 31 & 11 & -4 \\
12 & 35 & 11 & 10 \\
13 & 35 & 14 & 3 \\
14 & 31 & 16 & 8 \\
\hline Mean & 35.4 & 13.5 & 1.4 \\
SEM & 1.3 & 1.2 & 1.7 \\
\hline
\end{tabular}

MNI coordinates of tFUS focus center point for each subject. Related to Figure 1.

Table S2. ERP peak-to-peak amplitude at FCz comparing Sham and tFUS groups.

\begin{tabular}{|c|c|c|c|c|c|c|}
\hline \multirow[b]{2}{*}{ ERP complex } & \multicolumn{3}{|c|}{ Fear congruent } & \multicolumn{3}{|c|}{ Neutral congruent } \\
\hline & Sham & tFUS & $p$ & Sham & tFUS & $p$ \\
\hline D-N1 & $-3.28 \pm 0.55$ & $-5.28 \pm 0.62$ & $0.025^{*}$ & $-3.19 \pm 0.45$ & $-5.61 \pm 0.61$ & $0.003^{*}$ \\
\hline D-N1 - D-P1 & $5.49 \pm 0.90$ & $7.07 \pm 0.62$ & 0.36 & $4.96 \pm 0.94$ & $6.25 \pm 0.62$ & 0.31 \\
\hline D-P1 - T-N1 & $-5.73 \pm 1.14$ & $-7.79 \pm 0.55$ & 0.75 & $-6.57 \pm 1.07$ & $-6.89 \pm 0.60$ & 0.96 \\
\hline T-N1 - P2 & $3.39 \pm 0.45$ & $5.33 \pm 0.42$ & $0.007^{*}$ & $4.48 \pm 0.42$ & $4.83 \pm 0.68$ & $0.047^{*}$ \\
\hline $\mathrm{P} 2$ - N2 & $0.05 \pm 0.56$ & $-0.68 \pm 0.33$ & 0.44 & $-0.40 \pm 0.55$ & $-0.85 \pm 0.29$ & 0.19 \\
\hline \multirow[t]{2}{*}{$\mathrm{N} 2-\mathrm{P} 3$} & $0.68 \pm 0.39$ & $1.92 \pm 0.25$ & 0.007 & $0.20 \pm 0.47$ & $2.47 \pm 0.27$ & $0.003^{*}$ \\
\hline & \multicolumn{3}{|c|}{ Fear incongruent } & \multicolumn{3}{|c|}{ Neutral incongruent } \\
\hline ERP complex & Sham & tFUS & $p$ & Sham & tFUS & $p$ \\
\hline D-N1 & $-2.66 \pm 0.55$ & $-5.96 \pm 0.68$ & $0.001^{*}$ & $-2.75 \pm 0.70$ & $-6.17 \pm 0.60$ & $0.009^{*}$ \\
\hline D-N1 - D-P1 & $5.40 \pm 1.00$ & $7.22 \pm 0.64$ & 0.21 & $4.90 \pm 0.92$ & $6.67 \pm 0.61$ & 0.27 \\
\hline D-P1 - T-N1 & $-6.09 \pm 1.24$ & $-7.28 \pm 0.67$ & 0.69 & $-5.35 \pm 1.02$ & $-6.63 \pm 0.65$ & 0.52 \\
\hline T-N1 - P2 & $5.02 \pm 0.49$ & $5.68 \pm 0.55$ & 0.057 & $4.23 \pm 0.51$ & $5.67 \pm 0.58$ & $0.023 *$ \\
\hline $\mathrm{P} 2-\mathrm{N} 2$ & $-2.95 \pm 0.52$ & $-2.97 \pm 0.37$ & 0.56 & $-2.43 \pm 0.44$ & $-2.70 \pm 0.36$ & 0.86 \\
\hline N2 - P3 & $3.97 \pm 0.73$ & $4.34 \pm 0.68$ & 0.90 & $4.06 \pm 0.65$ & $4.78 \pm 0.50$ & 0.43 \\
\hline
\end{tabular}

Amplitude displayed as median \pm SEM $(\mu \mathrm{V})$ and represent peak-to-through (ERP complex) amplitude values from individual subjects' ERP peaks. $p$ - values represent the results of permutation testing across groups $(* p<0.05)$. Related to Figure 2 and Table S4. See also Table S3.

Table S3. ERP peak latency at FCz comparing Sham and tFUS groups.

\begin{tabular}{lllllll}
\hline & \multicolumn{3}{l}{ Fear congruent } & & & \multicolumn{3}{l}{ Neutral congruent } \\
ERP peak & Sham tFUS & $p$ & & Sham FUS & $p$ \\
\hline
\end{tabular}




\begin{tabular}{|c|c|c|c|c|c|c|}
\hline Distractor-N1 & $104 \pm 3$ & $98 \pm 2$ & 0.57 & $102 \pm 4$ & $98 \pm 2$ & 0.92 \\
\hline Distractor-P1 & $146 \pm 4$ & $148 \pm 2$ & 0.43 & $144 \pm 4$ & $148 \pm 2$ & 0.36 \\
\hline Target-N1 & $228 \pm 7$ & $222 \pm 7$ & 0.65 & $220 \pm 7$ & $222 \pm 6$ & 0.95 \\
\hline P2 & $340 \pm 4$ & $344 \pm 5$ & 0.10 & $340 \pm 4$ & $340 \pm 5$ & 0.75 \\
\hline $\mathrm{N} 2$ & $404 \pm 4$ & $396 \pm 6$ & 0.10 & $402 \pm 4$ & $374 \pm 5$ & $0.005^{*}$ \\
\hline \multirow[t]{2}{*}{ P3 } & $476 \pm 5$ & $484 \pm 6$ & 0.97 & $476 \pm 5$ & $484 \pm 8$ & 0.82 \\
\hline & \multicolumn{3}{|c|}{ Fear incongruent } & \multicolumn{3}{|c|}{ Neutral incongruent } \\
\hline ERP peak & Sham & tFUS & $p$ & $\underline{\text { Sham }}$ & tFUS & $p$ \\
\hline Distractor-N1 & $96 \pm 3$ & $94 \pm 2$ & 0.82 & $100 \pm 3$ & $92 \pm 2$ & 0.19 \\
\hline Distractor-P1 & $142 \pm 3$ & $144 \pm 2$ & 0.51 & $140 \pm 3$ & $140 \pm 2$ & 0.66 \\
\hline Target-N1 & $224 \pm 8$ & $234 \pm 5$ & 0.76 & $220 \pm 6$ & $226 \pm 5$ & 0.24 \\
\hline P2 & $342 \pm 5$ & $338 \pm 5$ & 0.62 & $340 \pm 6$ & $338 \pm 4$ & 0.43 \\
\hline N2 & $406 \pm 8$ & $384 \pm 5$ & 0.33 & $400 \pm 7$ & $388 \pm 5$ & 0.43 \\
\hline P3 & $486 \pm 5$ & $482 \pm 6$ & 0.27 & $486 \pm 5$ & $478 \pm 6$ & 0.36 \\
\hline
\end{tabular}

Latencies are displayed as median \pm SEM (ms). $p$ - values represent the results of permutation testing across groups $\left(^{*} p<\right.$ 0.05). Related to Figure 2. See also Table S2.

Table S4. ERP peak-to-peak amplitude at FCz compared within each group using non-parametric Friedman's test

\begin{tabular}{|c|c|c|c|c|c|c|}
\hline \multicolumn{7}{|l|}{ Sham } \\
\hline \multirow[b]{2}{*}{ ERP complex $\chi^{2}$} & \multirow[b]{2}{*}{$p$} & \multicolumn{5}{|l|}{ Post - hoc statics } \\
\hline & & $\begin{array}{l}\text { Neutral Fear } \\
\text { con vs.con } \\
\text { incon incon }\end{array}$ & $\begin{array}{r}\text { Con } \\
\text { vs.fear } \\
\text { neu } \\
\end{array}$ & $\begin{array}{c}\text { Incon } \\
\text { vs.fear } \\
\text { neu }\end{array}$ & $\begin{array}{c}\text { Fear } \\
\text { vs.vs. } \\
\text { incon } \\
\end{array}$ & $\begin{array}{l}\text { con } \\
\text { neuNeu con vs. } \\
\quad \text { fear incon }\end{array}$ \\
\hline
\end{tabular}




\begin{tabular}{|c|c|c|c|c|c|c|c|c|}
\hline D-N1 & 1.11 & 0.77 & - & - & - & - & - & - \\
\hline D-N1 - D-P1 & 7.63 & 0.054 & - & - & - & - & - & - \\
\hline D-P1 - T-N1 & 5.06 & 0.17 & - & - & - & - & - & - \\
\hline T-N1 - P2 & 13.46 & $0.004^{*}$ & 1 & $0.002^{*}$ & 0.64 & 0.34 & 0.47 & 0.24 \\
\hline P2 - N2 & 30.43 & $<0.001^{*}$ & 0.013 & $<0.001^{*}$ & 1 & 1 & $0.005^{*}$ & $<0.001^{*}$ \\
\hline N2 - P3 & 31.89 & $<0.001^{*}$ & $0.003^{*}$ & $<0.001^{*}$ & 1 & 0.86 & 0.05 & $<0.001^{*}$ \\
\hline \multicolumn{9}{|l|}{ tFUS } \\
\hline \multirow[b]{2}{*}{ ERP complex } & & & \multicolumn{6}{|c|}{ Post - hoc statistics } \\
\hline & $\chi^{2}$ & $p$ & $\begin{array}{l}\text { Neutral } \\
\text { con vs } \\
\text { incon } \\
\end{array}$ & $\begin{array}{c}\text { Fear } \\
\text { s.con } \\
\text { incon } \\
\end{array}$ & $\begin{array}{c}\text { Con } \\
\text { vs.fear } \\
\text { neutral } \\
\end{array}$ & \begin{aligned} \multicolumn{2}{r}{ Incon } \\
vs.fear v \\
l $\quad$ neutral \\
\end{aligned} & $\begin{array}{l}\text { Fear cc } \\
\text { vs.vs. ne } \\
\text { incon } \\
\end{array}$ & $\begin{array}{l}\text { con } \\
\text { heuNeu con vs. } \\
\text { fear incon } \\
\end{array}$ \\
\hline D-N1 & 10.37 & $0.016^{*}$ & 0.11 & 0.24 & 1 & 1 & $0.032^{*}$ & 0.644 \\
\hline D-N1 - D-P1 & 10.54 & $0.014^{*}$ & 1 & 0.86 & 1 & $0.032 *$ & 1 & $0.032^{*}$ \\
\hline D-P1 - T-N1 & 9.69 & $0.021^{*}$ & 1 & 1 & 0.17 & 0.17 & 0.24 & 0.11 \\
\hline T-N1 - P2 & 0.60 & 0.90 & - & - & - & - & - & - \\
\hline P2 - N2 & 22.29 & $<0.001^{*}$ & 0.12 & $<0.001^{*}$ & 1 & 1 & $0.008^{*}$ & $0.008^{*}$ \\
\hline N2 - P3 & 19.11 & $<0.001^{*}$ & $0.008^{*}$ & $0.020^{*}$ & 1 & 1 & $0.005^{*}$ & $0.032 *$ \\
\hline
\end{tabular}

Statistics from non-parametric Friedman's test performed across neutral congruent, fear congruent, neutral incongruent and fear incongruent trials, with three degrees of freedom for all tests, both groups $\mathrm{n}=14$. Post-hoc statics listed for each trial pair, all post-hoc $p$-values are Bonferroni-corrected. $\left({ }^{*} p<0.05\right)$. Abbreviations: Related to Figure 2 and Table S2. See also Table S3.

Table S5. Heart rate metrics compared across groups and time with presentation of emotion face distractors and tFUS stimulation.

\begin{tabular}{|c|c|c|c|c|c|c|c|c|c|c|c|c|}
\hline & \multicolumn{3}{|c|}{$\begin{array}{l}\text { Main effect } \\
\text { faces }\end{array}$} & \multicolumn{3}{|l|}{$\begin{array}{l}\text { Main } \\
\text { group }\end{array}$} & \multicolumn{3}{|c|}{$\begin{array}{l}\text { Interaction } \\
\text { group } \times \text { faces }\end{array}$} & \multicolumn{2}{|l|}{$\begin{array}{l}\text { Post-hoc } \\
\text { Sham }\end{array}$} & \multirow{2}{*}{$\begin{array}{l}\text { tFUS } \\
\% \\
\text { Change } p\end{array}$} \\
\hline & $\begin{array}{l}F(1,2 \\
5)\end{array}$ & $p$ & $\eta_{p^{2}}$ & $\begin{array}{l}F(1,2 \\
5)\end{array}$ & $p$ & $\eta_{p^{2}}$ & $\begin{array}{l}F(1,2 \\
5)\end{array}$ & $p$ & $\eta_{p^{2}}^{2}$ & $\begin{array}{l}\text { \% } \\
\text { Change }\end{array}$ & $p$ & \\
\hline \multirow{3}{*}{ HR (bpm) } & & & 0.0 & & 0.2 & 0.0 & & & 0.0 & & 0.04 & \\
\hline & 2.35 & 0.14 & 9 & 1.31 & 6 & 5 & 2.36 & 0.14 & 9 & $\uparrow 3 \pm 1 \%$ & $3^{*}$ & $0 \pm 2 \% 1.00$ \\
\hline & & & 0.1 & & 0.2 & 0.0 & & & 0.1 & & 0.02 & \\
\hline R-R (ms) & 2.74 & 0.11 & 0 & 1.63 & 1 & 6 & 2.91 & 0.10 & 0 & $\downarrow 3 \pm 1 \%$ & $8^{*}$ & $0 \pm 2 \% 0.97$ \\
\hline SDNN & & 0.006 & 0.2 & & 0.5 & 0.0 & & 0.023 & 0.1 & 2 & 0.00 & \\
\hline$(\mathrm{ms})$ & 9.12 & $*$ & 7 & 0.42 & 2 & 2 & 5.86 & $*$ & 9 & $\downarrow 0 \pm 4 \%$ & $1^{*}$ & $\downarrow 2 \pm 6 \% 0.67$ \\
\hline SD1 (ms) & 1.53 & 0.23 & 0.0 & 0.02 & 0.8 & 0.0 & 0.05 & 0.82 & 0.0 & $\downarrow 4 \pm 6 \%$ & 0.32 & $\downarrow 3 \pm 5 \% 0.48$ \\
\hline
\end{tabular}




\begin{tabular}{|c|c|c|c|c|c|c|c|c|c|c|c|}
\hline & & 6 & & 9 & 0 & & & 0 & & & \\
\hline & & $0.007 \quad 0.2$ & & 0.4 & 0.0 & & 0.023 & 0.1 & 2 & 0.00 & \\
\hline \multirow[t]{2}{*}{ SD2 (ms) } & 8.68 & * 6 & 0.64 & 3 & 3 & 5.87 & $*$ & 9 & $\downarrow 2 \pm 4 \%$ & $1^{*}$ & $\downarrow 2 \pm 7 \% 0.71$ \\
\hline & & 0.1 & & 0.8 & 0.0 & & & 0.0 & $\uparrow 2$ & 0.03 & \\
\hline \multirow[t]{2}{*}{ SD1/SD2 } & 3.42 & 0.0762 & 0.04 & 4 & 0 & 1.82 & 0.19 & 7 & $6 \pm 9 \%$ & $1^{*}$ & $\uparrow 3 \pm 6 \% 0.72$ \\
\hline & & $0.004 \quad 0.2$ & & 0.4 & 0.0 & & & 0.0 & & & 0.00 \\
\hline \multirow[t]{2}{*}{$\mathrm{LF}\left(\mathrm{ms}^{2}\right)$} & 9.83 & 8 & 0.58 & 5 & 2 & 0.74 & 0.40 & 3 & $\downarrow 6 \pm 4 \%$ & 0.13 & $\downarrow 1 \pm 4 \% 8^{*}$ \\
\hline & & $0.004 \quad 0.2$ & & 0.4 & 0.0 & & & 0.0 & $\uparrow$ & & 0.00 \\
\hline \multirow[t]{2}{*}{$\mathrm{HF}\left(\mathrm{ms}^{2}\right)$} & 9.83 & * 8 & 0.58 & 5 & 2 & 0.74 & 0.40 & 3 & $8 \pm 5 \%$ & 0.13 & $\uparrow 2 \pm 4 \% 8^{*}$ \\
\hline & & 0.0180 .2 & & 0.3 & 0.0 & & & 0.0 & & 0.24 & $1 \quad 0.02$ \\
\hline LF/HR & 6.41 & 0 & 0.93 & 4 & 4 & 0.67 & 0.42 & 3 & $\downarrow 8 \pm 8 \%$ & 5 & $\downarrow 8 \pm 7 \% 3^{*}$ \\
\hline
\end{tabular}

Mixed-measures RM-ANOVA on heart rate and HRV metrics collected during the final three minutes of baseline (simple flanker no faces, no stimulation), and the first three minutes of the main trail period (flanker arrows were displayed with emotional distractor faces in the background and the stimulation group received online tFUS on each trial). Related to Figure 6.

Table S6. Response accuracy does not differ across groups.

\begin{tabular}{|c|c|c|c|c|c|c|}
\hline \multicolumn{4}{|c|}{ Accuracy (\% correct) } & \multicolumn{3}{|c|}{ Mann-Whitney } \\
\hline Congruent & Sham & & tFUS & $p$ & $\mathbf{U}$ & $\mathbf{W}$ \\
\hline Baseline & 99.86 & $\pm 0.14 \%$ & $100.00 \pm 0.00 \%$ & 0.77 & 91.0 & 196.0 \\
\hline Fear & 98.36 & $\pm 0.60 \%$ & $97.71 \pm 0.67 \%$ & 0.60 & 109.5 & 214.5 \\
\hline Neutral & 98.07 & $\pm 0.45 \%$ & $96.43 \pm 1.22 \%$ & 0.84 & 102.5 & 207.5 \\
\hline Oddball & 99.21 & $\pm 0.79 \%$ & $96.29 \pm 1.77 \%$ & 0.33 & 119.5 & 224.5 \\
\hline Post & 99.57 & $\pm 0.23 \%$ & $98.97 \pm 0.75 \%$ & 0.95 & 99.5 & 204.5 \\
\hline \multicolumn{7}{|c|}{ Incongruent } \\
\hline Baseline & 100.00 & $\pm \quad 0.00 \%$ & $99.31 \pm 0.31 \%$ & 0.21 & 126.0 & 231.0 \\
\hline Fear & 90.86 & $\pm 1.58 \%$ & $85.00 \pm 2.62 \%$ & 0.11 & 133.0 & 238.0 \\
\hline Neutral & 90.70 & $\pm 1.69 \%$ & $85.71 \pm 2.05 \%$ & 0.09 & 135.0 & 240.0 \\
\hline Oddball & 93.32 & $\pm 2.96 \%$ & $95.71 \pm 1.83 \%$ & 0.70 & 89.0 & 194.0 \\
\hline Post & 99.40 & $\pm 0.26 \%$ & $99.22 \pm 0.78 \%$ & 0.40 & 79.0 & 184.0 \\
\hline
\end{tabular}

Group level statics (non-parametric Mann-Whitney tests) are listed on the right. Test statics performed on number of trials incorrect. See also Table 1.

Table S7. PANS scores.

\begin{tabular}{llll}
\hline PANAS Positive & \multicolumn{1}{l}{ Sham } & tFUS \\
\hline Baseline & $31.6 \pm 1.8$ & $30.4 \pm 2.0$ \\
Post & $25.9 \pm 2.6$ & $24.8 \pm 2.2$ \\
PANAS Negative & & & \\
\hline Baseline & $13.8 \pm 1.0$ & $13.0 \pm 0.8$ \\
Post & $13.5 \pm 1.1$ & $15.4 \pm 1.2$ \\
\hline PANAS Score for
\end{tabular}

PANAS Scores for each group (mean \pm SEM). 